\title{
Temporal-spatial structure of magnetic merging at the magnetopause inferred from 557.7-nm all-sky images
}

\author{
N. C. Maynard ${ }^{1}$, J. Moen ${ }^{2}$, W. J. Burke ${ }^{3}$, M. Lester $^{4}$, D. M. Ober ${ }^{1}$, J. D. Scudder ${ }^{5}$, K. D. Siebert ${ }^{1}$, D. R. Weimer ${ }^{1}$, \\ C. T. Russell ${ }^{6}$, and A. Balogh ${ }^{7}$ \\ ${ }^{1}$ ATK Mission Research, Nashua, New Hampshire, USA \\ ${ }^{2}$ University of Oslo, Oslo, Norway \\ ${ }^{3}$ Air Force Research Laboratory, Hanscom Air Force Base, Massachusetts, USA \\ ${ }^{4}$ University of Leicester, Leicester, UK \\ ${ }^{5}$ Department of Physics and Astronomy, University of Iowa, Iowa City, Iowa, USA \\ ${ }^{6}$ University of California at Los Angeles, Los Angeles, CA, USA \\ ${ }^{7}$ Imperial College, Exhibition Road, London, SW7 2BW, UK
}

Received: 18 September 2003 - Revised: 18 March 2004 - Accepted: 8 April 2004 - Published: 7 September 2004

\begin{abstract}
We demonstrate that high-resolution 557.7-nm all-sky images are useful tools for investigating the spatial and temporal evolution of merging on the dayside magnetopause. Analysis of ground and satellite measurements leads us to conclude that high-latitude merging events can occur at multiple sites simultaneously and vary asynchronously on time scales of $30 \mathrm{~s}$ to $3 \mathrm{~min}$. Variations of $557.7 \mathrm{~nm}$ emissions were observed at a $10 \mathrm{~s}$ cadence at Ny-Ålesund on 19 December 2001, while significant changes in the IMF clock angle were reaching the magnetopause. The optical patterns are consistent with a scenario in which merging occurs around the rim of the high-latitude cusp at positions dictated by the IMF clock angle. Electrons energized at merging sites represent plausible sources for $557.7 \mathrm{~nm}$ emissions in the cusp. Polar observations at the magnetopause have directly linked enhanced fluxes of $\geq 0.5 \mathrm{keV}$ electrons with merging. Spectra of electrons responsible for some of the emissions, measured during a DMSP F15 overflight, exhibit "inverted$\mathrm{V}$ " features, indicating further acceleration above the ionosphere. SuperDARN spectral width boundaries, characteristic of open-closed field line transitions, are located at the equatorward edge of the $557.7 \mathrm{~nm}$ emissions. Optical data suggest that with IMF $B_{Y}>0$, the Northern Hemisphere cusp divides into three source regions. When the IMF clock angle was $\sim 150^{\circ}$ structured $557.7-\mathrm{nm}$ emissions came from east of the 13:00 MLT meridian. At larger clock angles the emissions appeared between 12:00 and 13:00 MLT. No significant 557.7-nm emissions were detected in the prenoon MLT sector. MHD simulations corroborate our scenario, showing that with the observed large dipole-tilt and IMF clock angles,
\end{abstract}

Correspondence to: N. C. Maynard

(nmaynard@mrcnh.com) merging sites develop near the front and eastern portions of the high-altitude cusp rim in the Northern Hemisphere and near the western part of the cusp rim in the Southern Hemisphere.

Key words. Magnetospheric physics (auroral phenomena; solar wind-magnetosphere interactions; magnetopause, cusp and boundary layers)

\section{Introduction}

Ground-based observations are important because they allow the temporal evolution of phenomena to be followed. This is especially important for the study of reconnection, perhaps the most important process occurring on the magnetopause. This process is generally viewed only as a transient in spacecraft observations. If we could monitor the temporal behavior of reconnection over periods of hours, we could understand how merging evolves at any one location, how extensive that location may be, and how merging at multiple locations combines to provide the drivers for convective motions in the ionosphere and magnetosphere. In our earlier papers we showed that the fast responses of 557.7-nm emissions to changes in source properties in all-sky images of the cusp are ideal for following temporal and spatial variations in the dayside merging process (Maynard, 2003, 2004). We interpret these 557.7-nm signals as footprints of magnetopause merging. In a sense, these footprints provide "television" records of the temporal and spatial variability of interplanetary magnetic field (IMF) coupling to the magnetosphere. This paper (1) ties optical responses to variations in the IMF, (2) provides a plausible mechanism for this coupling, (3) places the 
557.7-nm observations in the context of SuperDARN radar and 630.0-nm optical observations, and (4) derives characteristics of the merging process based on the optical variations.

On 19 December 2001, 557.7-nm all-sky images were acquired at 10-s intervals at Ny-Ålesund. Characteristic variations in the optical measurements coincided with times when large variations in the IMF clock angle were expected to reach the magnetosphere. To reach and support the above conclusions, we begin by establishing the large geophysical context for understanding the Ny-Ålesund measurements. Solar wind and IMF measurements acquired near the first Lagrangian point (L1) by sensors on the Advanced Composition Explorer (ACE) spacecraft are compared with those of Cluster to estimate actual transport times to Earth. Particle and field measurements from sensors on two Defense Meteorological Satellite Program (DMSP) satellites are used to specify approximate locations of the prevailing large-scale convection pattern and morning/afternoon segments of cusp precipitation. Keogram representations of optical emissions detected at 557.7 and $630.0 \mathrm{~nm}$ by meridional scanning photometers at Ny-Ålesund between 09:10 and 10:10 UT establish the local context for the analysis of all-sky images taken near 09:15 UT and between 09:48 and 09:58 UT. Images from the latter period are compared with SuperDARN radar measurements from Pykkvibaer, Iceland and Hankasalmi, Finland, to establish correspondences with local plasma flows and spectral-broadening boundaries. A plausible tie between the $557.7 \mathrm{~nm}$ emissions and the merging process is introduced with non-concurrent Polar data and supported by the DMSP overflights. The discussion section integrates the present observations to establish their relationships with previously reported measurements and with predictions of the Integrated Space weather Model (ISM) (White et al., 2001). These conclusions draw on a number of recent results, which are first introduced below.

Maynard et al. (2001c) compared short-period wave structures measured in the upstream interplanetary magnetic field (IMF) with similar electric field variations detected by a sounding rocket launched from Ny-Ålesund, Svalbard into the dayside cusp region. Correlations observed during the rocket flight indicated that the lag time between the Lagrangian point and Earth was considerably less than the advection time. Such lag times require that, through IMF $B_{X}$, planes of constant phase for the interplanetary electric field (IEF) tilt with respect to the Y-Z plane. The analysis indicated that magnetic merging near the Southern Hemisphere cusp produced the observed Northern Hemisphere electrical field variations. Moreover, the sequence of brightened 557.7-nm emissions imaged by a high-resolution, all-sky camera matched ionospheric electric-field signatures of enhanced merging. Maynard et al. (2001c) also found that IMF $B_{Y}$ bifurcated the cusp so that features in the all-sky observations could be identified with separate Northern or Southern Hemispheres sources. They postulated that merging occurs around the rim of the cusp at locations wherever the draped IMF is nearly antiparallel to terrestrial field lines abutting the magnetopause.
Weimer et al. (2002) demonstrated a technique for determining variable lag times of signals propagating between satellites in the solar wind. Comparing lag times between ACE and three other satellites, they derived orientations of tilted phase planes and showed that the orientation could change dramatically within $10 \mathrm{~min}$. Lag times to magnetopause locations could be either longer or shorter than the advection times whenever IMF $B_{X}$ was a significant component. Calculating lag times correctly is critical for relating specific features in the upstream solar wind with dynamics observed in near-Earth space.

Maynard et al. (2003b) determined that whenever the IMF clock angle is $<150^{\circ}$, merging most often occurs at high latitudes near the cusp. Relevant data were acquired by Polar while skimming the magnetopause and by Cluster in the Northern Hemisphere cusp. SuperDARN monitored the ionospheric response. Both data and magnetohydrodynamic (MHD) simulations suggested that merging can move away from equatorial latitudes even when the IMF has a strong southward component if the dipole tilt angle is large.

Much of our present understanding of cusp optical morphology evolved from analyses of data captured by 630.0-nm meridian scanning photometers. Low-energy cusp electrons excite oxygen atoms into the metastable $\mathrm{O}\left({ }^{1} \mathrm{D}\right)$ state, whose lifetime for emitting a photon is $\sim 100 \mathrm{~s}$. Most $630.0-\mathrm{nm}$ emissions come from altitudes $>200 \mathrm{~km}$ where non-radiative collision rates are low. By contrast, the lifetime for atoms in the $\mathrm{O}\left({ }^{1} \mathrm{~S}\right)$ state to emit $557.7 \mathrm{~nm}$ photons is $\sim 1 \mathrm{~s}$. Rees (1963) showed that $1 \mathrm{keV}(0.5 \mathrm{keV})$ electrons lose most of their energy near $150 \mathrm{~km}(190 \mathrm{~km})$. Since energies of most electrons precipitating in the cusp are $<1 \mathrm{keV}$, most 557.7-nm emissions in the cusp are expected to originate in this range of altitudes. The relatively quick relaxation time of $\mathrm{O}\left({ }^{1} \mathrm{~S}\right)$ allows us to use $557.7-\mathrm{nm}$ responses to identify short time-scale variations of cusp sources (Maynard et al., 2001c; Maynard, 2003a, b).

Sandholt et al. $(1996,1998)$ described seven categories of 557.7- and 630.0-nm dayside auroral responses to varied orientations of the IMF. Categories 1 and 2 are dominated by $630.0-\mathrm{nm}$ emissions that occur during periods of southward and northward IMF, respectively. They have sharp boundaries collocated with the equatorward and poleward boundaries of cusp precipitation. Category 3 was associated with weak and diffuse 557.7-nm emissions caused by plasma sheet electrons precipitating equatorward of the cusp. Category 4 (5) and 6 (7) emissions occur on the dawn (dusk) edges of the cusp, for IMF conditions with northward and southward components, respectively. They often manifest "fan-arc" structures, characterized by relatively intense 557.7-nm emissions associated with electron precipitation from low-latitude boundary layers. Sandholt and Farrugia (2002) and Sandholt et al. (2002) found that, during transitions between northward and southward IMF, category 1 and 2 emissions may coexist for short periods. All-sky images show strong and variable 557.7-nm emissions on the poleward edge of the cusp during the period of northward IMF (Sandholt et al., 2002). 
The spectral widths of high frequency (HF) radar echoes detected by SuperDARN receivers broaden in the cusp region (Baker et al., 1995). The low-latitude edge of increased spectral width is often abrupt and is used as a dayside proxy to mark the equatorward boundary of cusp precipitation and the open-closed boundary (Baker et al., 1997). Rodger et al. (1995) and Moen et al. (2001a) located the spectral-width boundary within $1^{\circ}$ latitude of the cusp boundary. Due to time-of-flight effects the spectral-width boundary may be displaced poleward of the open-closed boundary (Pinnock and Rodger, 2001). Chisham et al. (2002a) showed that in the Southern Hemisphere during an IMF $B_{Y}<0$ interval the prenoon spectral-width boundary was $\sim 2^{\circ}$ poleward of its postnoon location. They attributed the difference to two separate reconnection sites located in opposite hemispheres. Tied to the distant merging site, plasma flow near the morning sector boundary appeared to be nearly steady. The displacement of that boundary was from convection during the time-of-flight from the distant source. Large line-of-sight flow bursts characterized the afternoon boundary that was tied to the nearhemisphere merging site. The pre- and post-noon regions were distinct and separated. In another event, sunward convection observed between the two regions confirmed the predictions of Coleman et al. (2001) for antiparallel merging at solstice (Chisham et al., 2002b). It should be noted that the precise determination of the boundary between large and small spectral widths can be difficult. An assessment of the accuracy in determining such a boundary can be found in Chisham and Freeman (2003).

\section{Measurement techniques}

Ground-based optical instruments at Ny-Ålesund on the island of Spitzbergen in the Svalbard archipelago included meridian-scanning photometers recording emissions at 630.0 and $557.7 \mathrm{~nm}$. The scanning direction was near the magnetic meridian on a line between $\mathrm{Ny}$-Ålesund and Longyearbyen $\left(78.2^{\circ} \mathrm{N}, 15.7^{\circ} \mathrm{E}\right)$. A meridian scan was made every $20 \mathrm{~s}$. All-sky photometric measurements were recorded every $10 \mathrm{~s}$, recording $557.7-\mathrm{nm}$ images for 2 out of every three intervals and 630.0-nm emissions in the third interval. Integration times for each observation were $5 \mathrm{~s}$, with the assigned time being at the beginning of the measurement. Thus, allsky measurements at $557.7 \mathrm{~nm}$ were available with basically a 10-s repetition rate while images at $630.0 \mathrm{~nm}$ were made every $30 \mathrm{~s}$. The all-sky images of the 557.7-nm $(630.0-\mathrm{nm})$ emissions have been projected onto a sphere at 150 or $190 \mathrm{~km}$ $(220 \mathrm{~km})$ and superposed onto a geographic (geomagnetic) map.

Dayside SuperDARN radar data (Greenwald et al., 1995) have been used to identify the cusp by relating an increase in the spectral width of the return signals with crossing the open-closed boundary (e.g. Baker et al., 1995; Chisham et al., 2002a, b). Like the optical data, SuperDARN provides a two-dimensional picture of the dayside dynamics when return echoes are strong enough to be detected. With 16 beam directions and up to 70 range gates along each beam, lineof-sight ion drifts can be determined over a large region. On 19 December 2001, the CUTLASS pair, located at Hankasalmi, Finland, and Pykkvibaer, Iceland, was operated on 2-min scans with an integration time of $7 \mathrm{~s}$ along each beam. The Hankasalmi radar was swept from east to west while the Pykkvibaer radar was swept from north to south. The times associated with the radar plots are the beginning times of the sweeps.

DMSP satellites are three-axis stabilized spacecraft that fly in circular, sun-synchronous polar orbits (inclination $98.8^{\circ}$ ) at an altitude of $840 \mathrm{~km}$. The geographic local times of the orbits are either near the 18:00-06:00 LT (F 11, F13) or 21:00-09:00 LT (F12, 14, 15) meridians. We present observations from F13 and F15. Due to offsets between the geographic and geomagnetic poles DMSP satellites sample wide ranges of magnetic local times over the course of a day. The ascending nodes of DMSP orbits are on the dusk side of the Earth. Thus, the satellites move toward the northwest (southeast) in the evening (morning) sector. Each satellite carries a suite of sensors to measure (1) fluxes of auroral particles (SSJ4), and (2) densities, temperatures and drift motions of ionospheric ions and electrons (SSIES). All of the satellites except F11 carry magnetometers (SSM) to monitor perturbations of the Earth's magnetic field.

Identical SSJ4 sensors are mounted on the top surfaces of DMSP satellites to measure fluxes of down-coming electrons and ions in 19 logarithmically spaced energy steps between $30 \mathrm{eV}$ and $30 \mathrm{keV}$ (Hardy et al., 1984). Unfortunately, fluxes of ions with energies below $1 \mathrm{keV}$ are unavailable from the F15 satellite. SSIES consist of a spherical Langmuir probe mounted on a $76-\mathrm{cm}$ boom to measure the densities and temperatures of ambient electrons and three separate sensors mounted on a conducting plate facing in the ram direction. These are: 1) an ion trap to measure the total ion density, 2) an ion drift meter to measure the horizontal ( $\mathrm{VH})$ and vertical (VV) cross-track components of plasma drifts, and 3) a retarding potential analyzers (RPA) to measure ion temperatures and in-track components of plasma drift $\mathrm{V}_{\| v}$, (Rich and Hairston, 1994). RPA current-voltage sweeps also indicate relative concentrations of heavy $\left(\mathrm{O}^{+}\right)$and light $\left(\mathrm{H}^{+}\right.$, $\mathrm{He}^{++}$) ions. Ion drift meter data are combined with spacecraft ephemeris and model magnetic field values to calculate the in-track component of electric fields. These are then integrated along the trajectory to estimate distributions of electric potential.

SSM sensors are triaxial fluxgate magnetometers that are mounted on the bodies of the F12-F14 spacecraft. For DMSP flights 15 and higher, magnetometers are mounted on 5-m booms, making them less susceptible to spacecraftgenerated contamination than their body-mounted predecessors. Magnetic field components were sampled at rates of $12 \mathrm{~s}^{-1}$ and are presented in a satellite-centered coordinate system. The $\mathrm{X}$ and $\mathrm{Y}$ axes point downward and along the spacecraft velocity, respectively. The Z-axis completes a right-hand coordinate system. Positive $\mathrm{Z}$ generally points in the antisunward direction. Data are presented as differences 
between measured values and those assigned by the IGRF-90 magnetic field model $\left(\delta B_{X}, \delta B_{Y}, \delta B_{Z}\right)$. Near-in-time observations from the SSM, SSIES and SSJ4 sensors on the F13 and F15 spacecraft are used to corroborate and interpret optical and radar measurements.

The ACE spacecraft monitors interplanetary conditions while flying in a halo orbit around the $L_{1}$ point in front of the Earth. At the time of the measurements ACE was located near $(X, Y, Z)_{G S E}=(234,25,11) \mathrm{R}_{E}$. The solar wind velocity was measured by the Solar Wind Electron, Proton, and Alpha Monitor (SWEPAM) (McComas et al., 1998). A tri-axial fluxgate magnetometer measured the interplanetary magnetic field vector (Smith et al., 1998).

At the time of interest on 19 December 2001, the Cluster spacecraft were located in the postnoon magnetosheath on the flank near GSE location $(5.5,18.3,-1.2) \mathrm{R}_{E}$. Magnetic field measurements were made on each of the Cluster spacecraft by tri-axial fluxgate magnetometers (Balogh et al., 2001). We use measurements from Cluster 1 to determine the lag time from ACE to the magnetosheath.

In 2001 Polar's apogee $\left(9 \mathrm{R}_{E}\right)$ was near the equator. As a consequence, for long intervals in March-April the orbit skimmed from south to north, along the dayside magnetopause. Polar closely followed magnetopause responses to temporal changes of the IMF and solar-wind pressure. Several sensors on Polar are used in this study. The Hydra Duo Deca Ion Electron Spectrometer (DDIES) (Scudder et al., 1995) consists of six pairs of electrostatic analyzers looking in different directions to acquire high-resolution energy spectra and pitch-angle information. High-resolution distributions of electrons with energies between $1 \mathrm{eV}$ and $10 \mathrm{keV}$ and ions with energies per charge ratio of $10 \mathrm{eV} \mathrm{q}^{-1}$ to $10 \mathrm{keV} \mathrm{q}^{-1}$ are provided every $2.3 \mathrm{~s}$. The electric field instrument (EFI) (Harvey et al., 1995) uses a biased double probe technique to measure vector electric fields from potential differences between 3 orthogonal pairs of spherical sensors. This paper presents measurements from the long wire antennas in the satellite's spin plane. The Magnetic Field Experiment (MFE) (Russell et al., 1995) consists of two orthogonal triaxial fluxgate magnetometers mounted on nonconducting booms. The electric and magnetic fields were sampled 40 and $20 \mathrm{~s}^{-1}$, respectively. Data presented in this paper were spin averaged using a least-squares fit to a sine function. Because of the orbital constraints, which keep Polar from sampling the cusp in December, measurements presented are from April 2001, and are not simultaneous with the other measurements presented.

\section{The integrated space weather prediction model (ISM)}

In the MHD simulations described here, ISM operates within a warped cylindrical computational domain whose origin is at the center of the Earth. Its domain extends $40 \mathrm{R}_{E}$ sunward, and $300 \mathrm{R}_{E}$ anti-sunward in direction, and $60 \mathrm{R}_{E}$ radially from the Earth-Sun line. The cylindrical domain has an interior spherical boundary approximately located at the bottom of the E-layer $(100 \mathrm{~km})$. The cylindrical to spherical interface is at $3 \mathrm{R}_{E}$, which is pure spherical out to $3.5 \mathrm{R}_{E}$ and warps to pure cylindrical at large distances.

ISM uses standard MHD equations augmented by hydrodynamic equations in the collisionally-coupled thermosphere. Conceptually, as one moves toward the Earth, equations transition seamlessly from pure MHD in the solar wind and magnetosphere to those proper to the low-altitude ionosphere/thermosphere. The simulations discussed here contain specifically selected parameters and simplifying approximations. Finite-difference grid resolution varies from a few hundred kilometers in the ionosphere to several $\mathrm{R}_{E}$ near the computational domain's outer boundary. At the magnetopause, resolution ranges from 0.2 to $0.8 \mathrm{R}_{E}$. Explicit viscosity in the plasma momentum equation was set to zero. To approximate non-linear magnetic reconnection the explicit resistivity coefficient $v$ in Ohm's Law equation is zero if current density normal to $B$ is less than $3.16 \cdot 10^{-3} \mathrm{~A} \mathrm{~m}^{-2}$. In regions with perpendicular current densities above this threshold, $v=2 \cdot 10^{10} \mathrm{~m}^{2} \mathrm{~s}^{-1}$. In practice, this choice of $v$ leads to non-zero explicit resistivity near the subsolar magnetopause, and in the nightside plasma sheet. Dissipation, where needed to maintain numerical stability, is based on the partial donorcell method (PDM) as formulated by Hain (1987).

\section{Observations}

The global context for this December event is provided by the IMF, DMSP, and the meridian scanning photometer data in the first three subsections. In Sects. 4.4, 4.5 and 4.7 we present the 557.7-nm optical images and their associations with the global perspective through direct correlation. Comparisons with SuperDARN observations are made in Sect. 4.6. In Sect. 4.8 we show Polar observations which were made at the magnetopause in April 2001, to suggest the source of the 557.7-nm emissions. Section 4.9 uses a direct over-flight of DMSP to tie this suggestion to the optics. Interpretation and synthesis is provided in the Discussion (Sect. 5).

\subsection{IMF measurements}

Figures 1b-1d show the three components of the IMF measured by ACE (black traces) between 07:00 and 09:00 UT on 19 December 2001. During the periods of interest the average solar wind density and speed were approximately $2.5 \mathrm{~cm}^{-3}$ and $420 \mathrm{~km} / \mathrm{s}$, respectively. Using the ACE velocity, the advection times between the positions of ACE and Cluster were determined (between 57 and $63 \mathrm{~min}$ ) and plotted in Fig. 1a (green trace). The advection time was calculated without consideration of the additional delay imposed by crossing the bow shock ( $2-3 \mathrm{~min})$. As mentioned above, Weimer et al. (2002) showed that lag times are variable and may differ significantly from advection times, especially in the presence of a significant $B_{X}$. Owing to the dipole tilt and $B_{X}$, interaction times may not be the same for 
the two hemispheres (Maynard et al., 2001c). The Weimer et al. (2002) technique was used to calculate the variable lag times between ACE and Cluster (red trace in the top panel). Lag times ranged between 65 to $72 \mathrm{~min}$ in the interval of interest. The Cluster magnetic field data were then time adjusted to the ACE position by the variable lag and overlaid over the ACE data. The Cluster magnetic field values have been multiplied by 0.27 to accommodate the amplitude increase, which was experienced by crossing the bow shock into the magnetosheath. Negative $B_{X}$ combined with a dipole tilt away from the Sun suggests that the first interaction between the IMF and the magnetosphere occurred in the Southern Hemisphere. Positive IMF $B_{Y}$ indicates that merging can be expected on the dawn/dusk side of the southern/northern cusp.

Since the IMF clock angle is preserved across the bow shock (Song et al., 1992; Maynard et al., 2003b), it is a sensitive indicator for determining the orientation of the IMF that couples to the magnetosphere-ionosphere, thereby constraining possible merging locations. We use clock angles to estimate proper lags from ACE to the ionosphere. The insert in Fig. 1a plots the observed clock angle at ACE used in the comparisons below. The heavy black lines are meant to direct the reader's attention to changes in the clock angle observed at ACE that are rapid near 08:00 UT and from 08:30 to 08:50 UT, and slow near 08:15 UT. These intervals are the focus of the observations presented below.

The coupling time from the merging sites to the northern ionosphere depends on whether merging proceeds at high latitudes near the local or conjugate cusp, or near the sub-solar magnetopause. We fine-tune estimates of the interaction times from multiple sites in the discussion. Cluster was in the magnetosheath near GSE location $(5.5,18.3,-1.2) \mathrm{R}_{E}$. The delay to this flank location appears similar to the delay to the cusp ionosphere. We associate a 557.7-nm optical brightening observed near 09:55 UT with a clock angle turn to $180^{\circ}$ near $08: 46: 30$ UT. At this time the lag to Cluster was increasing from 65 to $70 \mathrm{~min}$. We approximate the lag time from ACE to Earth during this clock angle variation as $69.5 \mathrm{~min}$. Earlier optical variations near 09:10 UT are related to the IMF with a 71-min lag.

\subsection{DMSP measurements}

On 19 December 2001 the F13 and F15 satellites passed close to Ny-Ålesund during the period of optical data collection. Figures $2 a$ and $2 b$, respectively, display particle and plasma measurements from F15 and F13 as functions of UT, MLat, and MLT. The magnetic coordinates are at the satellite location within the International Geomagnetic Reference Field (IGRF-1990) model updated for secular change. The top two panels of each show directional differential fluxes of electrons and ions with energies between $30 \mathrm{eV}$ and $30 \mathrm{keV}$ in a standard energy-versus-time color spectrogram format. The ion sensor on F15 responsible for measuring ions with energies $\leq 1 \mathrm{keV}$ did not perform as planned, and these measurements should be ignored. Traces in the bottom panel in-

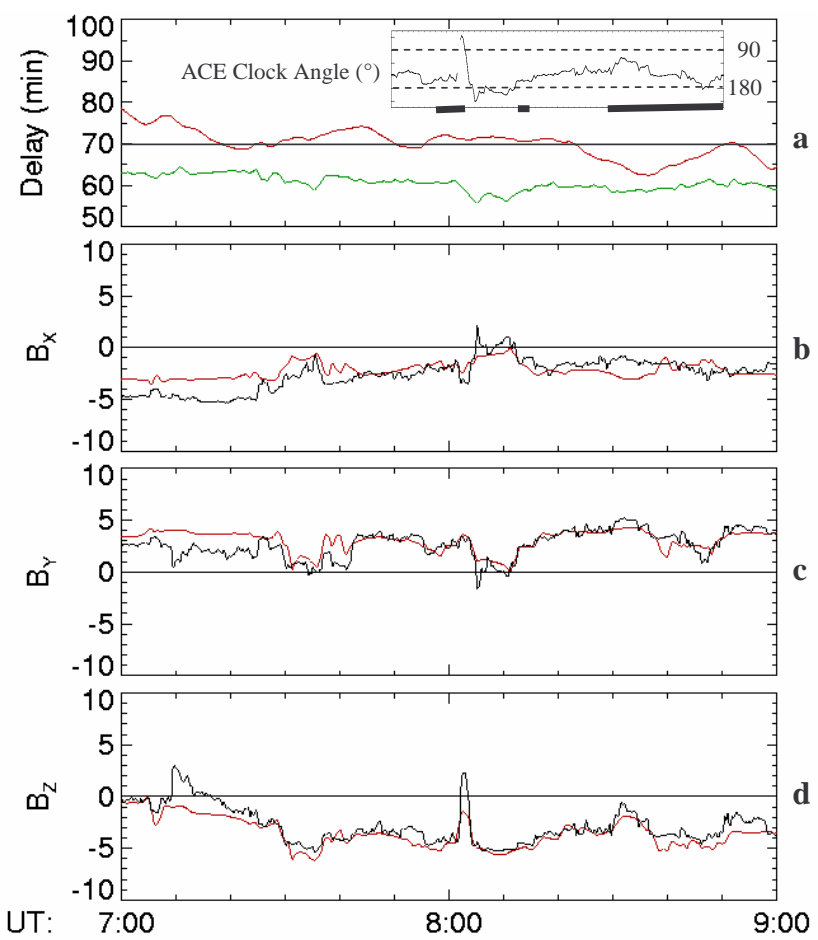

Fig. 1. Comparison of IMF data from ACE and magnetosheath data from Cluster to determine the applicable lag time between the satellites. (a) shows the advection delay (green) between ACE and Cluster calculated using the time-dependent ACE velocity. The variable lag using the technique of Weimer et al. (2002) is marked in red. $(\mathbf{b}-\mathbf{d})$ show three components of the IMF measured by ACE (black) for this interval. The corresponding Cluster magnetometer data, time adjusted according to the calculated variable lag, are overlaid in red. Because Cluster was located in the magnetosheath, the Cluster data have been multiplied by a factor of 0.27 to adjust for the increase in magnitude from passage through the bow shock. The insert in (a) shows the IMF clock angle at ACE for the period of interest. This clock angle plot is used in subsequent figures that compare the optical measurements with the IMF. The three intervals to be discussed are highlighted by the black bars.

dicate horizontal (purple) and vertical (green) drifts. Positive horizontal flows indicate convection with sunward components. The polar plots to the right of the spectrograms show MLat- vs. MLT-trajectories of the DMSP satellites during the UT periods of the spectrograms. The directions of horizontal plasma flows from the drift meter and the approximate location of $\mathrm{Ny}-$ Ålesund are indicated by arrows and the letter $\mathrm{N}$, respectively.

Looking first at Fig. 2a, we see that F15 crossed the auroral oval in the dusk sector between 09:16 and 09:19 UT. This region was characterized by fluxes of accelerated electrons with energies of several $\mathrm{keV}$ and by sunward plasma convection. Between 09:19 and 09:22 UT, F15 detected weak or sporadic electron fluxes, no higher energy ion fluxes and plasma flows $<1 \mathrm{~km} / \mathrm{s}$. However, between 09:23 and 09:25 UT, F15 observed intensified electron and ion fluxes, as well as a burst of strong $(>1 \mathrm{~km} / \mathrm{s})$ westward convection. 


\section{A $\quad$ F15 19 Dec 2001}

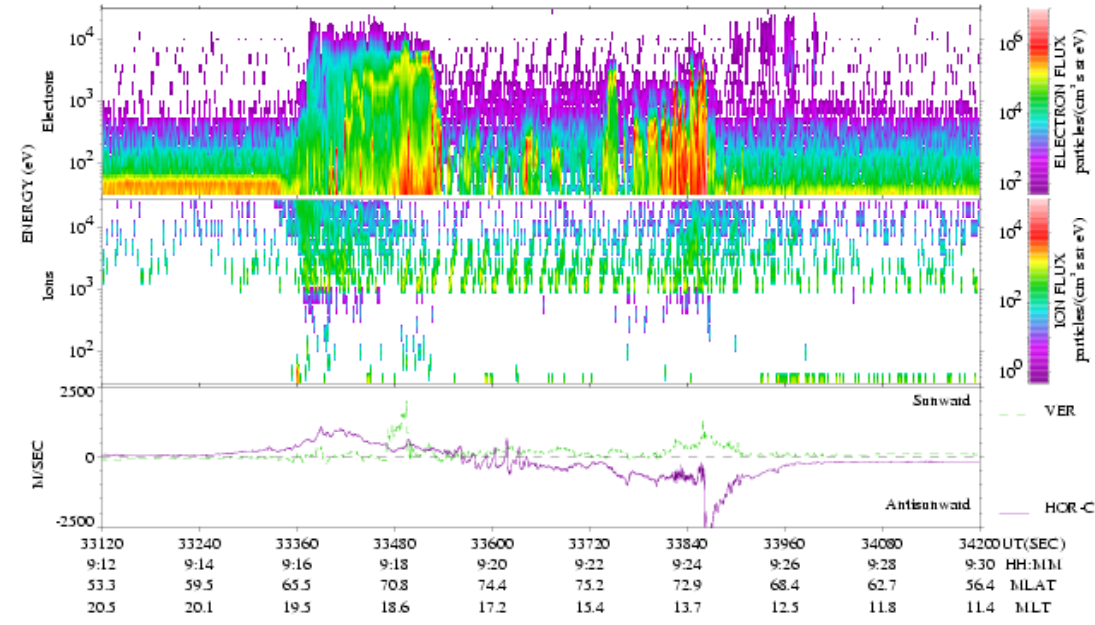

\section{B F13 19 Dec 2001}

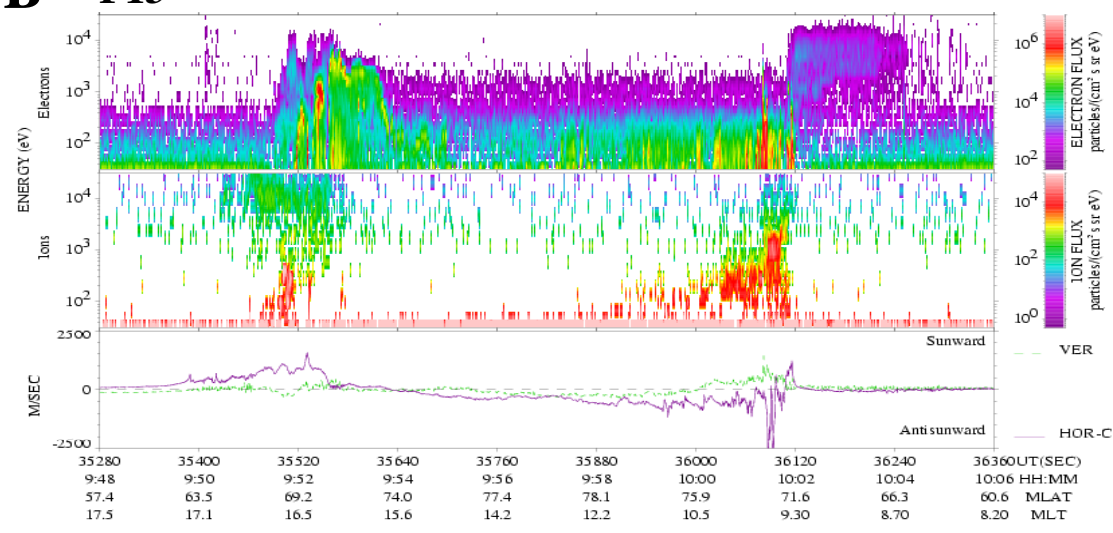

DMSP F15 12
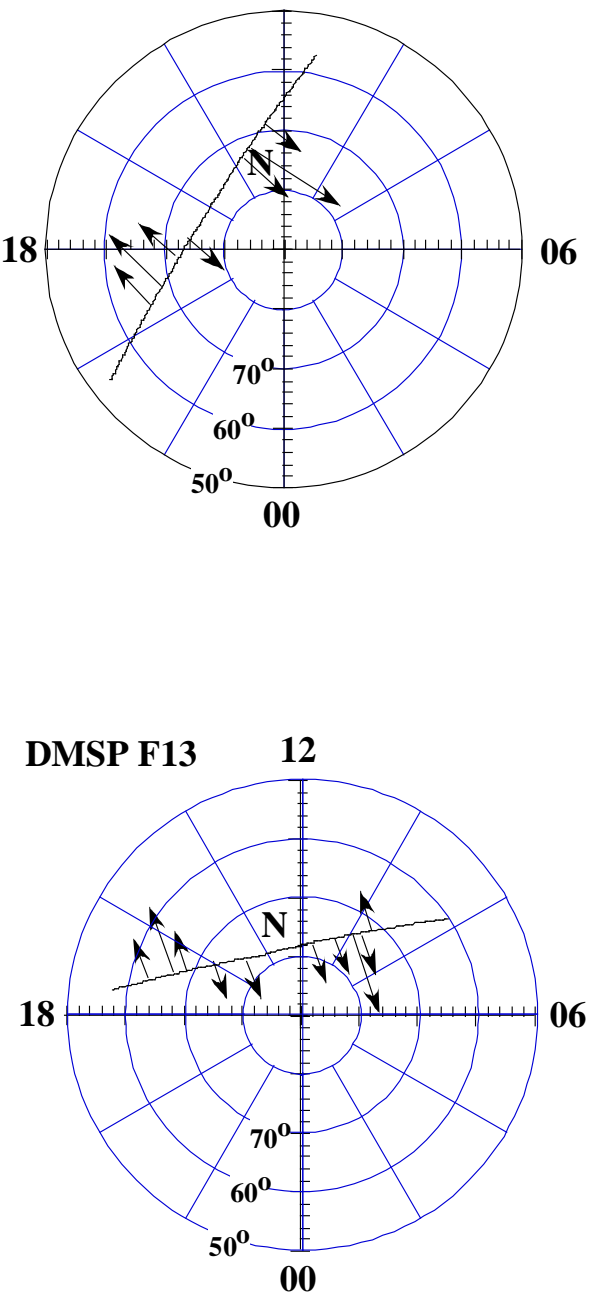

Fig. 2. DMSP F13 and F15 measurements including spectrograms for (a) electrons, (b) ions, and (c) horizontal and vertical drifts. At the right of each data set, the polar dial indicates the trajectory. The afternoon auroral zone crossing is later in local time than the cusp in each case. F13 remains poleward of cusp and mantle fluxes until just before crossing the morning side cusp near 09:30 MLT. The horizontal drift is strongest on the morning side as expected for this positive IMF $B_{Y}$ condition. Cusp fluxes are seen near 10:00 MLT just before the convection reversal. F15 skimmed the post noon cusp region observing bursts of electrons between 13:00 and 14:00 MLT. Note that the ion sensor on this spacecraft did not function below $1 \mathrm{keV}$.

Attention is directed to the two small inverted-V structures, which occurred between 09:24:00 and 09:24:27 UT (better visible in the expanded plot in Fig. 15). Peak energies of $\sim 2 \mathrm{keV}$ are consistent with the electrons having undergone field-aligned acceleration. Their locations near $72.9^{\circ}$ MLat and $13.7^{\circ}$ MLT places these events to the south and east of $\mathrm{Ny}$-Ålesund. Integration of the equivalent along-track electric field to determine the potential distribution along the spacecraft trajectory shows that F15 remained in the afternoon convection cell throughout the period of interest. A potential minimum of $-47.2 \mathrm{kV}$ was measured at MLat $=75.3^{\circ}$ and MLT=17.1.

F13 crossed the dayside ionosphere about a half hour after the F15 pass. The afternoon auroral oval near 16:00 MLT is remarkably similar to that seen by F15 near 19:00 MLT. The convection reversal at $74.7^{\circ}$ MLat and 15.60 MLT corresponds to a potential minimum of $-44.7 \mathrm{kV}$. The F13 trajectory passed to the north of Ny-Ålesund through a region of polar rain and antisunward convection. Beginning at $\sim 10: 00$ UT, F13 successively encountered fluxes of mantle ions, cusp ions/electrons, low-latitude boundary layer (LLBL) electrons and dayside plasma sheet electrons. Note that cusp electrons show no spectral signs of having undergone field-aligned acceleration. The cusp and LLBL fluxes were collocated with bursts of antisunward and sunward plasma drifts, respectively. Integration of the in-track component of electric field indicates that this reversal occurred at a potential maximum of $4.7 \mathrm{kV}$. This small positive potential 

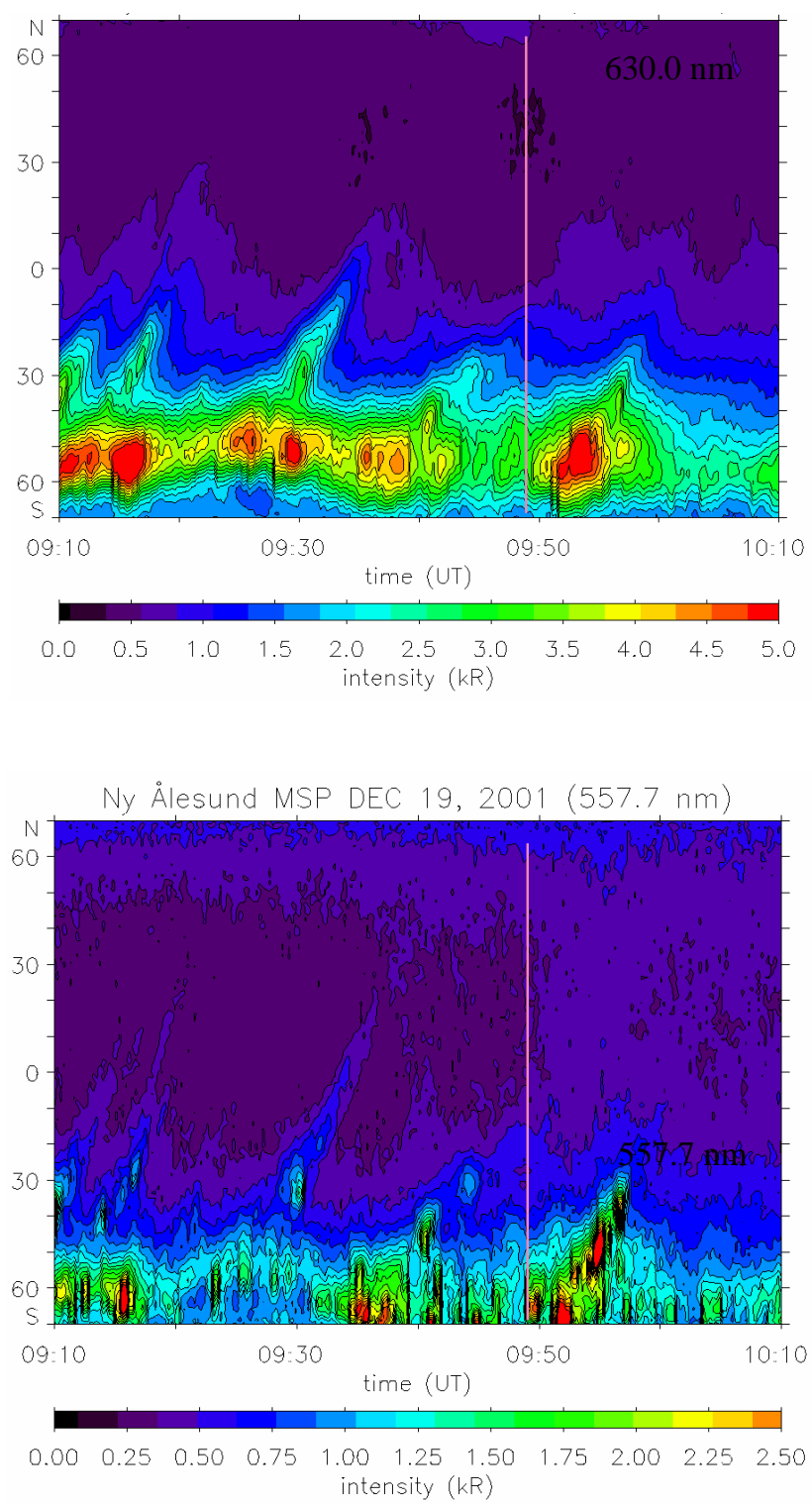

Fig. 3. Meridional scanning photometer measurements at 630.0 (top) and $557.7 \mathrm{~nm}$ (bottom) acquired between 09:10:00 and 10:10:00 UT on 19 December 2001.

suggests that F13 crossed only a small portion of the morning convection cell to which cusp particles have access. The stability of the large-scale electrodynamics detected by F13 and F15 in the high-latitude ionosphere is consistent with the variable IMF $B_{Y}>0$ and $B_{Z}<0$ conditions observed by ACE after 08:10 UT, but with a dominant clock angle $\sim 135^{\circ}$ (Fig. 1).

\subsection{Meridian scanning photometer measurements}

Figure 3 provides an overview of the optical dynamics over the period of interest as 630.0-nm (top) and 557.7-nm (bottom) emissions observed between 09:10 and 10:10 UT by magnetic-meridian scanning photometers (MSP) at Ny-Ålesund. All 630.0-nm emissions fall into category 1, as cataloged by Sandholt et al. (1998). Category 1 emissions generally have a sharp lower border and are characteristic of IMF orientations with negative $B_{Z}$. Dynamic cusp features evident in $630.0 \mathrm{~nm}$ data have been previously referred to as poleward moving auroral forms (PMAFS) (Vorobjev et al., 1975; Sandholt et al., 1986; Fasel, 1995). Such structures are typically seen for clock angles between $90^{\circ}$ and $140^{\circ}$ (Sandholt et al., 2004). They appear as a-periodic emission structures that move poleward with convection from lower latitude initiation points. Several 557.7-nm features are seen with three exceeding intensities $>2 \mathrm{kR}$. The strongest begin near 09:50 UT and are MSP signatures of optical structures presented in the all-sky images of Figs. 4-9. Of importance to this study is the fact that MSP data provide records of largescale intensifications at the local time of the station. All-sky images simultaneously cover several hours of local time. We argue that the 557.7-nm all-sky images resolve spatial and temporal details of interaction processes.

\subsection{All-sky imager measurements}

High-resolution 557.7-nm all-sky images acquired at Ny-Ålesund on 19 December 2001, were usually recorded at a 10 -s cadence. However, for every third image the imager shifted to observe at $630.0 \mathrm{~nm}$, leaving gaps in the 10-s record. Figure 4 shows a sequence of the 557.7-nm images, starting at 09:48:10 UT. The emissions have been projected to $190 \mathrm{~km}$ and overlaid on a geographic map. The white arrow in the upper left image points toward magnetic north. Vertical auroral rays in all-sky pictures may appear as emissions along a line pointing to magnetic zenith, located just below the center of the image. The auroral emissions on the dusk (right) side of these images, which are elongated toward magnetic zenith, can be interpreted as rayed forms with the footprints near the edge or just outside of the image. Red, yellow and pink fiducials have been placed at the edge of each image to help the reader follow temporal changes at particular locations. Each fiducial is roughly oriented toward magnetic zenith, and a circle or square appears at the base of fiducial lines to indicate enhanced emissions.

In the early part of this sequence emissions near the red and yellow fiducials were weak. At 09:49:30 UT emissions intensified near the yellow fiducial. After 09:51:00 UT they weakened and then alternated between small enhancements and weakenings during the remainder of Fig. 1. Emissions first appeared near the pink fiducial at 09:51:10 UT. Emissions also strengthen near the red fiducial from 09:50:00 to $09: 51: 40$ UT. In fact, a smaller enhancement appeared at 09:49:00 UT in line with the red fiducial at the northern edge of the emissions and away from the edge of the field of view. Some of the early emissions near the red fiducial originate just outside the field of view. Following the conceptual diagram of Maynard et al. (2001c), we believe that multiple merging sites became active on the magnetopause for varying intervals during this 4.5 -min sequence.

Figure 5 continues the sequence, covering the 09:53:00 to 09:57:30 UT interval. Emissions essentially vanished near 


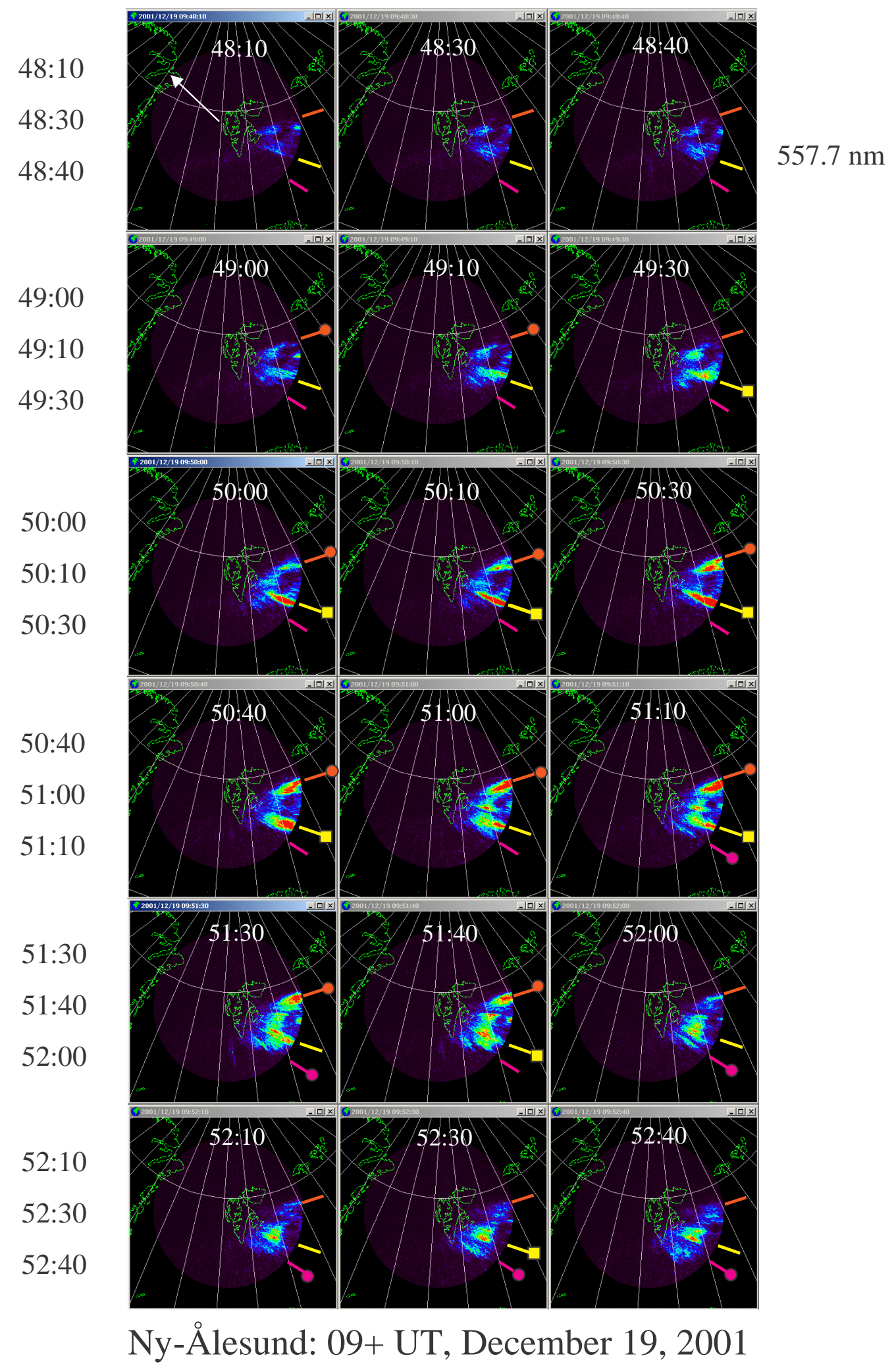

Fig. 4. All-sky images of 557.7-nm emissions over Ny-Ålesund taken at a cadence of 10s on 19 December 2001, from 09:48:10 to 09:52:40 UT. Occasionally, intervals are missing while other wavelengths were sampled. The images are mapped to $190 \mathrm{~km}$ on a geographic grid. Magnetic north is approximately in the direction of the white arrow in the upper left image. Red, yellow, and pink fiducials have been added at the same location in each image to guide the eye. Squares or circles indicate that a region is active. 


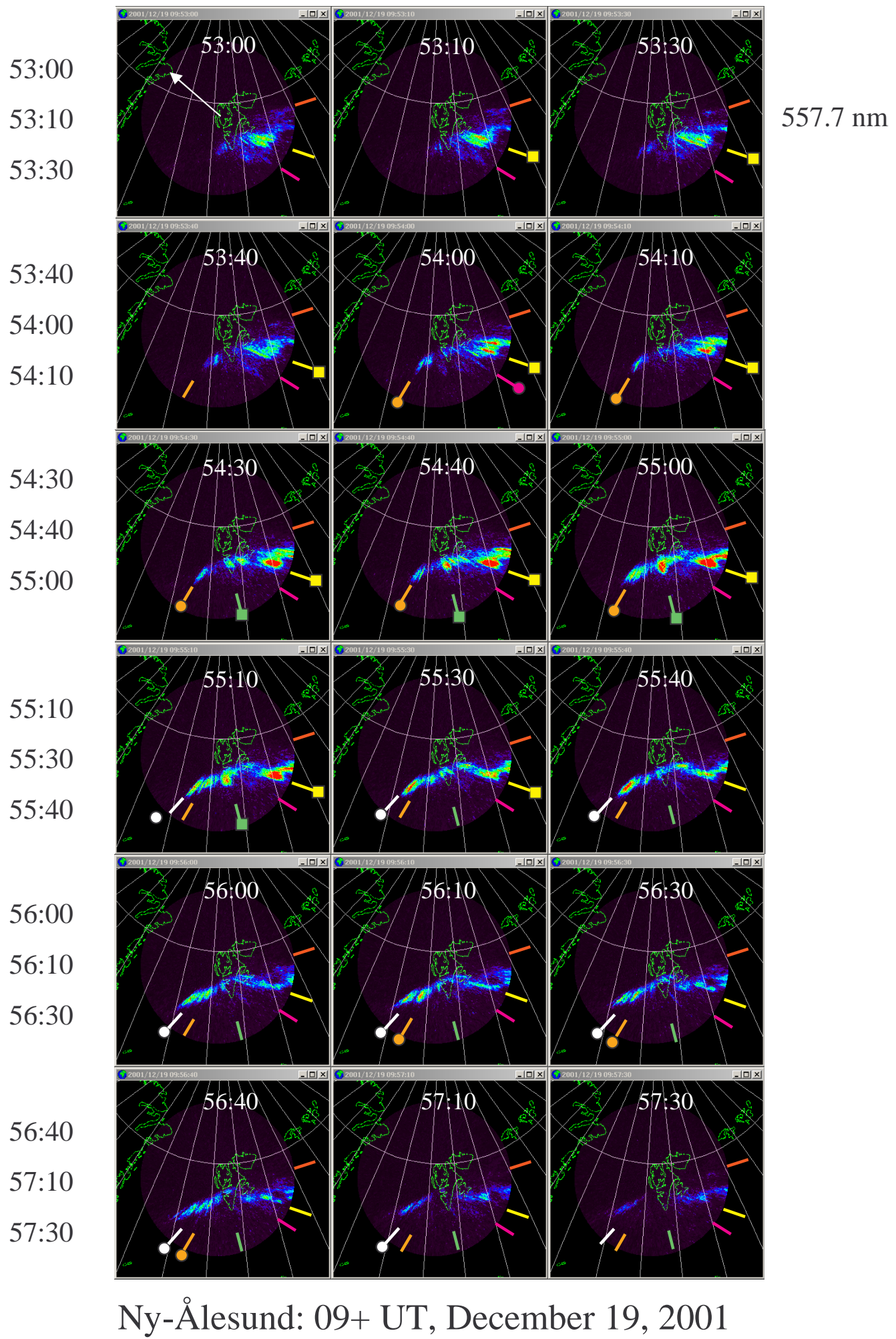

Fig. 5. 557.7-nm all-sky images continuing the sequence in Fig. 4 for the interval 09:53:00 to 09:57:30 UT. Green, orange, and white fiducials have been added along with those of Fig. 4 . 


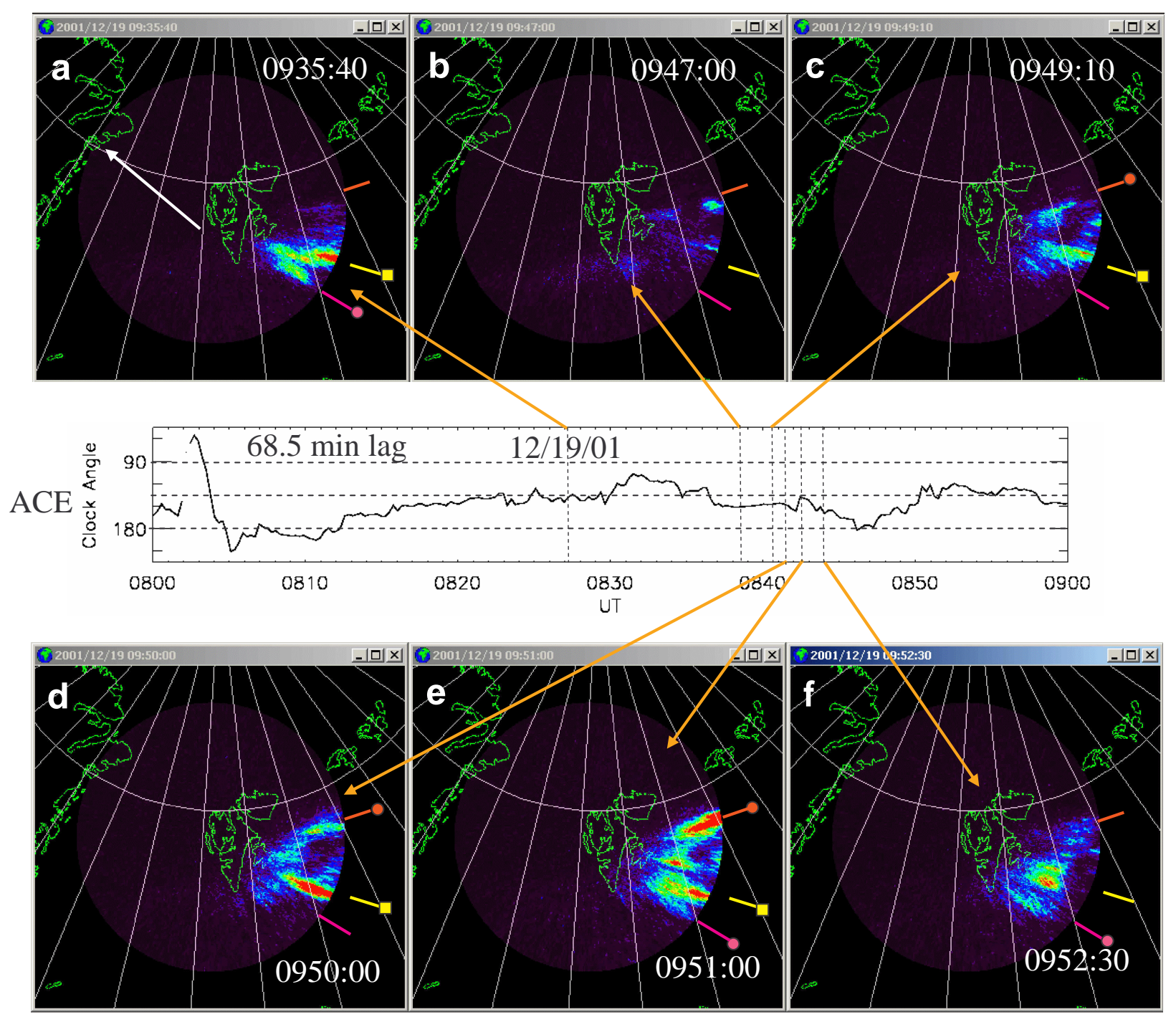

Ny-Ålesund: $557.7 \mathrm{~nm}$ - mapped to $190 \mathrm{~km}$

Fig. 6. Six 557.7-nm all-sky images from Ny-Ålesund between 09:35:40 and 09:53:30 UT mapped to 190 km. The IMF clock angle is displayed in the center panel with the UT of the ACE measurements. We associate each image with the IMF clock angle trace using a 69.5-min lag time. The orange arrows couple each image to the trace. Fiducials of Fig. 4 appear where appropriate.

the red fiducial. However, emissions came and went near the yellow fiducial and in the vicinity of three new fiducials (green, orange, and white), located to the magnetic south and west of Ny-Ålesund. Between 09:54:00 and 09:55:10 UT emissions appeared near the orange fiducial. For the next two minutes dominant emissions came from near the white fiducial. A 40-s enhancement developed near the magnetic meridian of Ny-Ålesund (green fiducial). By the time of the last few images, emissions faded at all locations. Over the 9-min interval spanned by Figs. 4 and 5, 557.7-nm emissions underwent dramatic changes in the location and intensity. In the roughly three hours of MLT sampled by the images, there is no location where emissions were constant.

Between 08:35 and 08:50 UT, ACE observed a rapid change in the IMF clock angle (Fig. 1). Dipole tilt interacts with $B_{X}$ to set the interaction times in each hemisphere, which may be unequal (Maynard et al., 2001c). The negative $B_{X}$ and the tilt away from the Sun of the dipole in this case mean that the first interaction of the IMF with the magnetosphere will be in the Southern Hemisphere. The positive $B_{Y}$ places the interaction on the dawn side in the south and on the dusk side in the north. The IMF clock angle is preserved across the bow shock near the nose (Song et al., 1992; Maynard et al., 2003b). It becomes a sensitive indicator of the proper lag in the magnetosheath, thereby defining the orientation of the IMF that couples into the magnetosphere-ionosphere system through merging. As shown below, we associate optical variations presented in Figs. 4 and 5 with changes in the IMF clock angle. Coupling times from the merging site to the northern ionosphere 

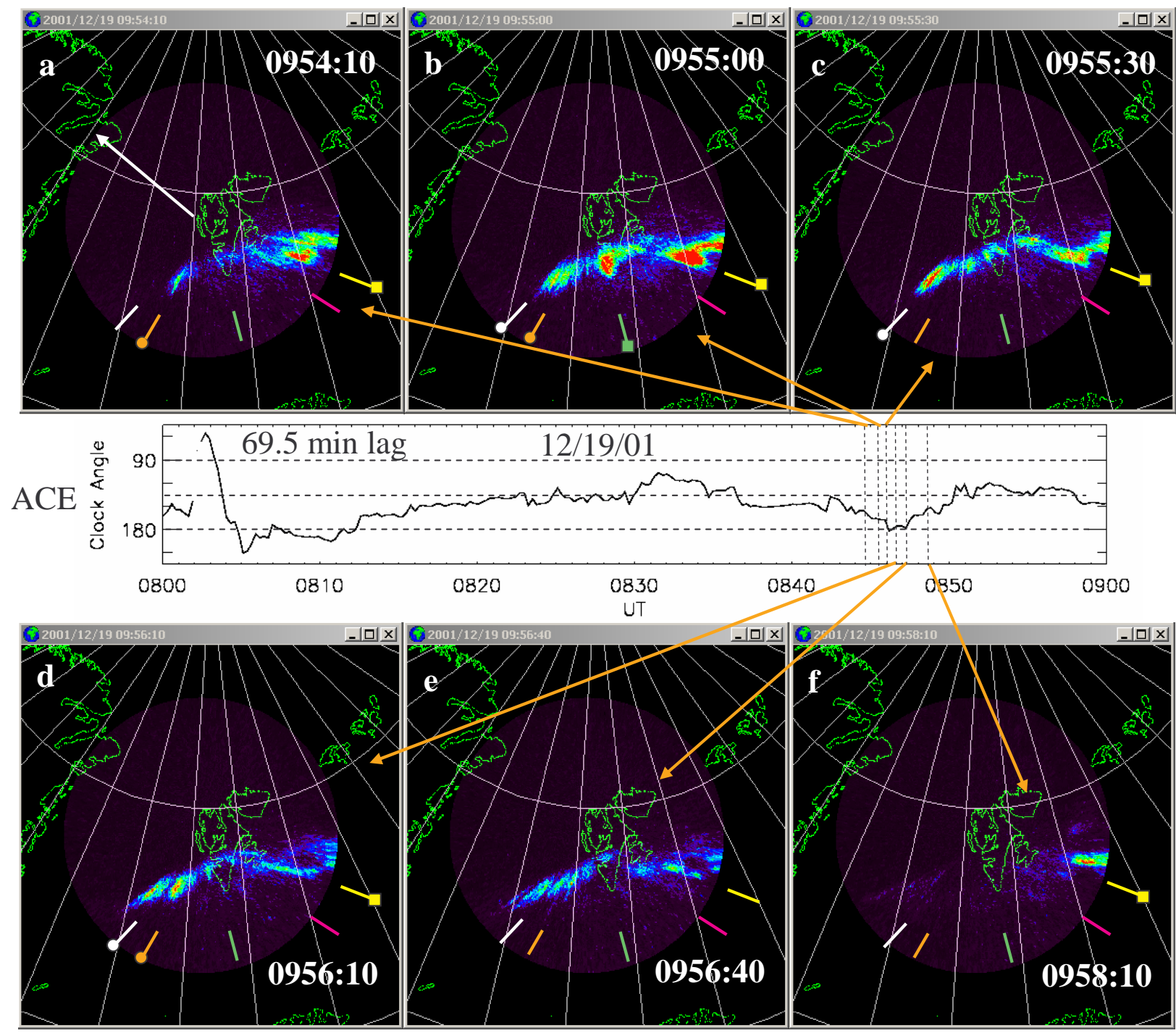

Ny-Ålesund: $557.7 \mathrm{~nm}$ - mapped to $190 \mathrm{~km}$

Fig. 7. Continuation of the sequence in Fig. 6 with six more images related to the ACE IMF clock angle with the 69.5-min lag. Fiducials of Fig. 5 appear where appropriate.

depend on whether the interaction occurs near the local cusp, the sub-solar magnetopause, or the conjugate cusp.

Figure 6 presents six 557.7-nm images and a plot of clock angles measured by ACE. The white arrow in the upper left image points to magnetic north. We associate changes in the clock angle observed at ACE just after 08:35 UT with changes in the emissions using an effective lag time of 68.5 to $69.5 \mathrm{~min}$ for the period encompassed in Figs. 4 and 5 . This lag time appears reasonable when compared to the 65 to $69 \mathrm{~min}$ lag to Cluster in the dusk magnetosheath (Figure 1b). A lag of $68.5 \mathrm{~min}$ has been used for the examples in Fig. 6. The orange arrows and vertical dashed lines couple images with the applicable IMF features at this lag. Fiducials added to the images are at the same locations as Figs. 4 and 5. The yellow fiducial marks the primary location for emissions of the sequence when the clock angle was between $135^{\circ}$ (dashed line) and $160^{\circ}$. Emissions increase at the red fiducial between 09:50:50 and 09:51:40 UT as illustrated in Fig. 6e. This coincides with a clock angle decrease to $\sim 135^{\circ}$. In clock-angle excursions $>155^{\circ}$, emissions began near the pink fiducial (Figs. 6e and 6f).

Our comparison with the IMF clock angles continues in Fig. 7 using a lag of $69.5 \mathrm{~min}$. In Fig. 7a (09:54:10 UT) emissions appeared near the orange fiducial to the magnetic west of Ny-Ålesund while the clock angle approached $180^{\circ}$. As the clock angle briefly decreased from $180^{\circ}$, the emissions appeared near the green fiducial (Fig. 7b), slightly to the west of Ny-Ålesund's magnetic meridian. Figures $7 \mathrm{c}$ and $7 \mathrm{~d}$ illustrate emissions near the white fiducial further to the west of Ny-Ålesund as the clock angle returned to $180^{\circ}$. In Fig. 7 e the clock angle rotated back to $160^{\circ}$. The 

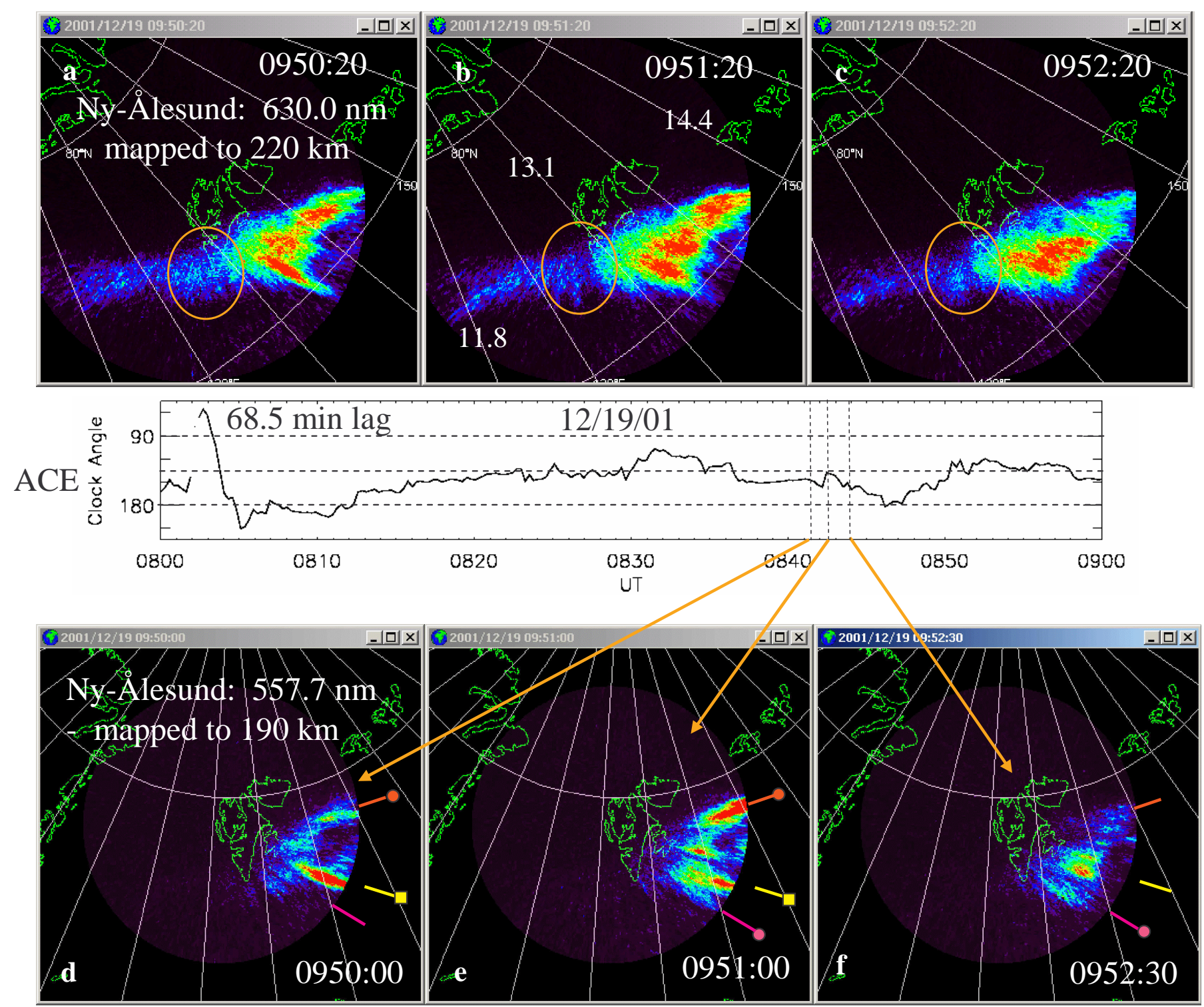

Fig. 8. 630.0-nm images (top) compared with the corresponding 557.7-nm images (bottom). The 557.7-nm images are the same as those in Figs. 6d, 6e, and 6f. The 630.0-nm images are in magnetic coordinates with the central meridian passing through Ny-Ålesund and two other meridians labeled with MLT. The orange circles in the top three images mark breaks in emissions between source regions.

clock angle reached $155^{\circ}$ in the last image with $557.7-\mathrm{nm}$ emissions mostly near the yellow fiducial.

Maynard et al. (2001c) suggested that the merging maximizes near the edge of the high-altitude cusp at locations that depend on IMF clock angles. Figures 4-7 clearly illustrate how the locations of the 557.7-nm emissions closely follow clock angle variations. These data also show that multiple locations can be active at the same time. With clock angles $\leq 150^{\circ}$, emissions came from the east of $\mathrm{Ny}$-Ålesund. At larger clock angles source locations moved toward earlier magnetic local times. If we equate variations in the intensities and locations of 557.7-nm emissions with changes in rates and locations of merging on the magnetopause, we can follow the dynamics of IMF coupling to the magnetosphere that drives ionospheric convection. In what follows we attempt to solidify this interpretation by relating the optical observations to other data sets, by continuing the comparison to other intervals, and by offering a plausible cause for the emissions.

\subsection{Comparisons with $630.0 \mathrm{~nm}$ emissions}

Figure 8 compares 557.7-nm and 630.0-nm emissions from the last three intervals of Fig. 6. The 630.0-nm images are projected to $220 \mathrm{~km}$ and overlaid with a dipole magnetic coordinate system centered on the magnetic pole with the central longitudinal line connecting with magnetic zenith at 

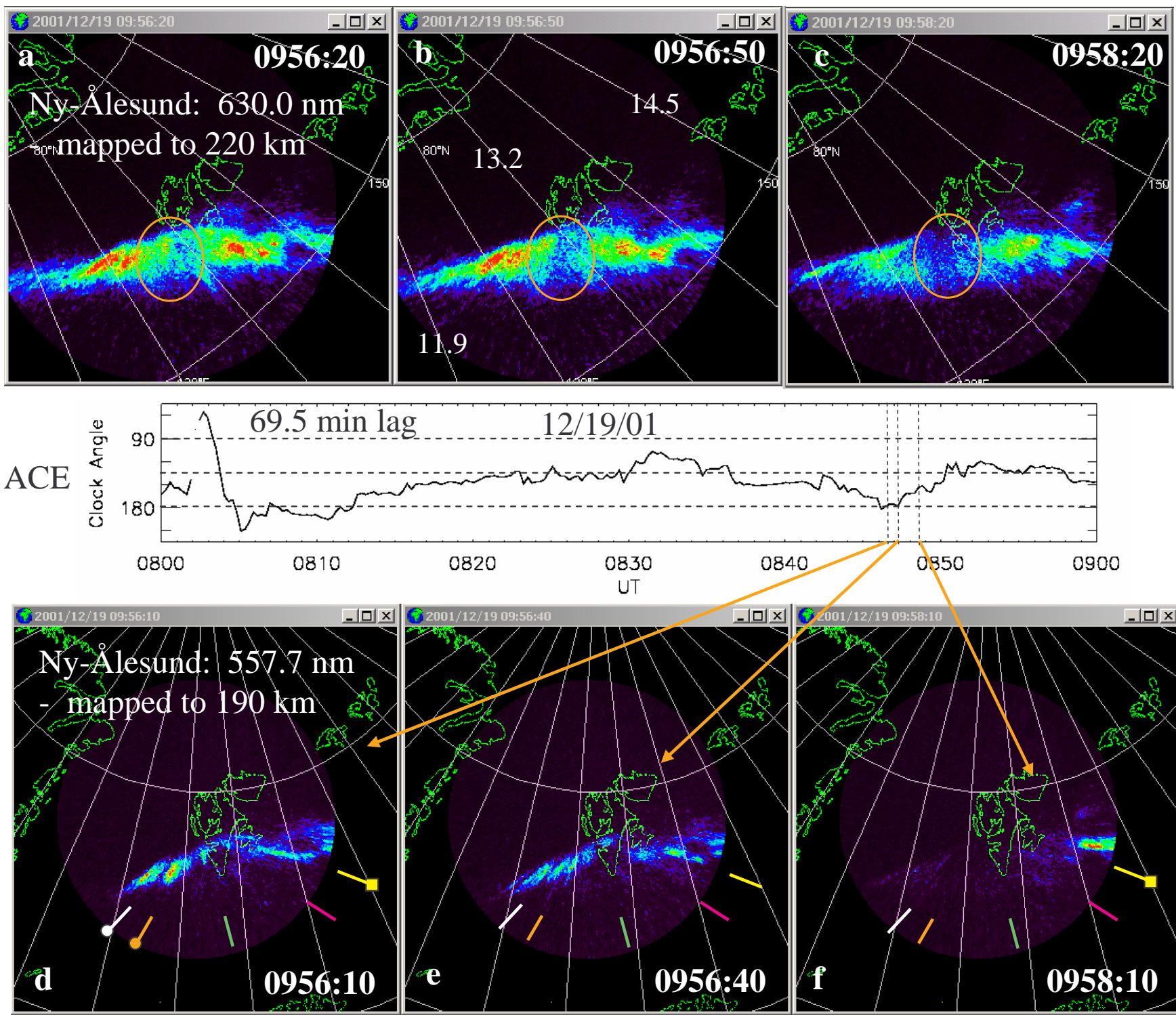

Fig. 9. Continuation of the 630.0- and 557.7-nm emission comparisons for times in Figs. 7d, 7e, and 7f. See caption for Fig. 8.

Ny-Ålesund. Longitudinal lines are marked every $10^{\circ}$ and are labeled with magnetic local time. Emissions detected after 13:00 MLT would be categorized as category 5 or 7 in the classification of Sandholt et al. (1998). Figure 9 displays 630.0- and 557.7-nm images for the last three intervals of Fig. 7. The emissions before 13:00 MLT in Figs. 9a, 9b, 9d, and $9 \mathrm{e}$ would be classified category 1 . In all the 630.0-nm images in Figs. 8 and 9 there appears to be a change in character just to the west of the $\mathrm{Ny}$-Ålesund magnetic meridian near 13:00 MLT. Orange circles highlight this feature. Figures $8 \mathrm{~b}, 8 \mathrm{c}$ and $9 \mathrm{c}$ show distinct separations or bifurcations of the regions. The region to the east of Ny-Ålesund extends to lower magnetic latitudes and in general has stronger emissions. This characteristic is modified in Figs. 9a and 9b, illustrating the interval when the IMF clock-angle was near $180^{\circ}$. In Fig. 9a, a bifurcation is not obvious. However, emissions at both wavelengths, from west of $\mathrm{Ny}$-Ålesund, are at higher latitudes than those from the east. Note that the detailed structuring of 557.7-nm emissions are less evident in the 630.0-nm images. This reflects a spatial smearing due to the long lifetime of the $\mathrm{O}\left({ }^{1} \mathrm{D}\right)$ state. As anticipated, the 630.0-nm emissions extend in magnetic local time over much more of the cusp region. We clearly associate the 557.7-nm emissions with the cusp derived from 630.0-nm observations (e.g. Sandholt et al., 1998). However, the tie to merging as the direct source observations of 557.7-nm emissions from east of 13:00 MLT places the cusp morphology in a new light. We return to this point in the discussion below. 
Dec.19, 2001: 0952

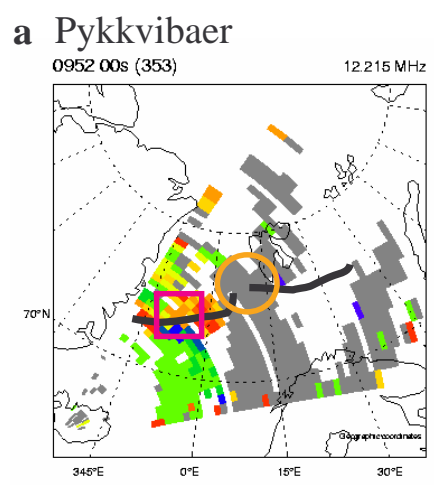

b Hankasalmi

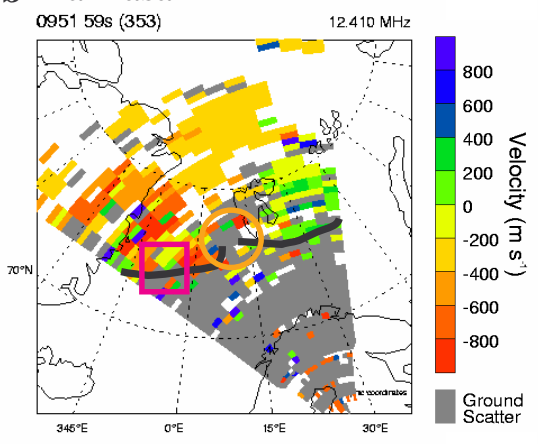

Ny-Ålesund 557.7 nm

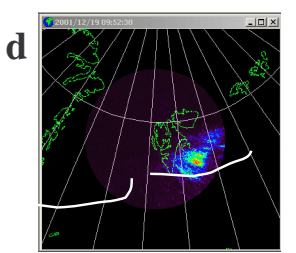

$150 \mathrm{~km}$

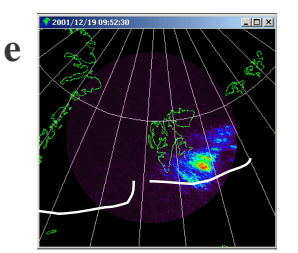

$190 \mathrm{~km}$

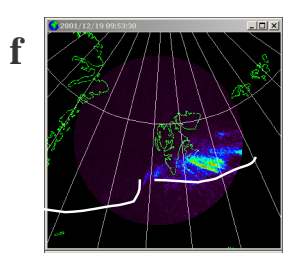

$190 \mathrm{~km}$ c Hankasalmi

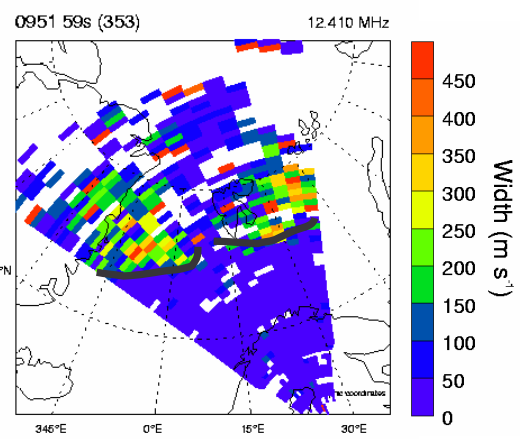

0952:30 UT

0952:30 UT

0953:30 UT

Ny-Ålesund 630.0 nm

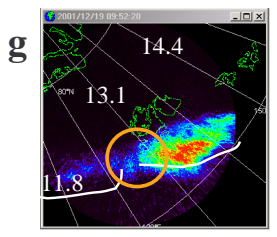

$220 \mathrm{~km}$

0952:20 UT

Fig. 10. Comparison of SuperDARN and optical data for the 2-min interval starting at 09:52 UT showing the line-of-sight velocities from (a) Pykkvibaer, Iceland, and (b) Hankasalmi, Finland, and the spectral widths (c) of the Hankasalmi signals. The Hankasalmi spectralwidth boundary has been determined and overlaid on the velocity plots. It appears in two segments because of the significant differences in magnetic latitude and the region of near null velocities in the break. The orange circle straddles the break and is at the same place as in the $630.0 \mathrm{~nm}$ optical data of Figs. 8 and 9. The red square highlights a region where in the previous several minutes there had been intense velocity toward Pykkvibaer. 557.7-nm all sky images are projected to (d) $150 \mathrm{~km}$ and (e) $190 \mathrm{~km}$ and overlaid on a geographic map. To emphasize the changing 557.7-nm images, two times are presented in (e) and (f) projected to $190 \mathrm{~km}$. $630.0 \mathrm{~nm}$ images (g) projected to $220 \mathrm{~km}$ are overlaid on a geomagnetic coordinate map. Magnetic local times are noted for three meridians. The spectral width boundaries are overlaid as white lines.

\subsection{SuperDARN measurements}

Figure 10 is a composite of the radar and optical data for the two-minute period starting at 09:52 UT. Figures 10a and $10 \mathrm{~b}$ show the line of sight velocity distributions detected at Pykkvibaer and Hankasalmi, respectively. Figure 10c plots the spectral width determined by the Hankasalmi radar. Figures $10 \mathrm{~d}, 10 \mathrm{e}, 10 \mathrm{f}$ and $10 \mathrm{~g}$ depict the all-sky data on comparable scale plots. How well the plots compare depends in part on the accuracy of our assumptions about the altitudes of the source data. The 557.7-nm emissions are projected to altitudes of both 150 and $190-\mathrm{km}$ to capture the range of optical responses due to differences in energies of source electrons. Figures 10e and 10f show the $190 \mathrm{~km}$ projections of the 557.7-nm data at 09:52:30 and 09:53:30 UT to illustrate the variability of the local dynamics during the 2-min radar scan. The 630.0-nm data were projected to $220 \mathrm{~km}$ and are displayed in MLat-MLT coordinates. The MLT of three meridians at the times of observations are noted. Our best estimate of the boundary between regions of high and low spectral width as determined in Fig. 10c, is overlaid on the other panels (as white lines on the all-sky figures). Note that the spectral-width boundary is split into two parts, reflecting toward and away bifurcations of ion drift measurements and a region where echoes are weak to non-existent. The split occurs in the region where the optical properties of $630.0 \mathrm{~nm}$ emissions change, as marked by orange circles in Figs. 8 and 9, as well as in Fig. 10. The bifurcation between the east and west branches is striking in Fig. 10b.

The SuperDARN boundaries match the equatorward boundary of optical emissions quite well. The plasma velocity observed at Hankasalmi is toward the radar on the east side of the pattern and away on the west. This is consistent with the dusk-dawn flow in the cusp expected under positive $B_{Y}$ IMF conditions. At Pykkvibaer in the region straddling the western boundary segment (red box) the flow is mixed. Two minutes earlier the flow in that region was strongly westward. Strong away flow also straddles this boundary in this region at Hankasalmi. The flow components from both radars combine to indicate a dusk-dawn flow or a poleward 
Dec.19, 2001: 0954

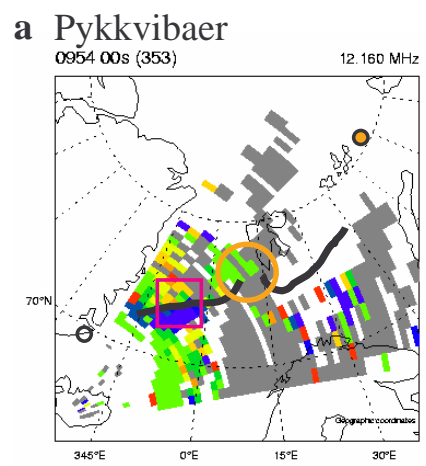

b Hankasalmi

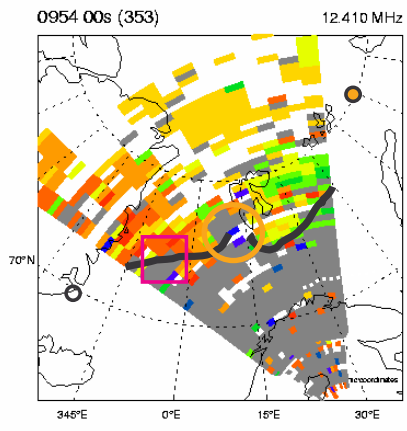

Ny-Ålesund 557.7 nm

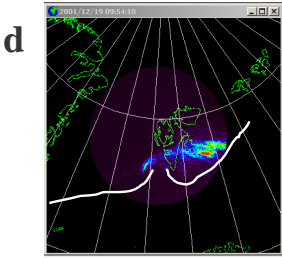

$150 \mathrm{~km}$
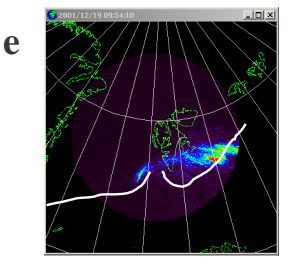

$190 \mathrm{~km}$

0954:10 UT

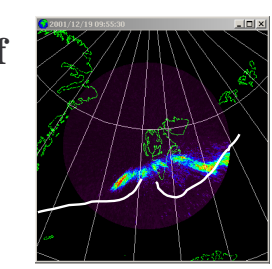

$190 \mathrm{~km}$

0955:30 UT c Hankasalmi
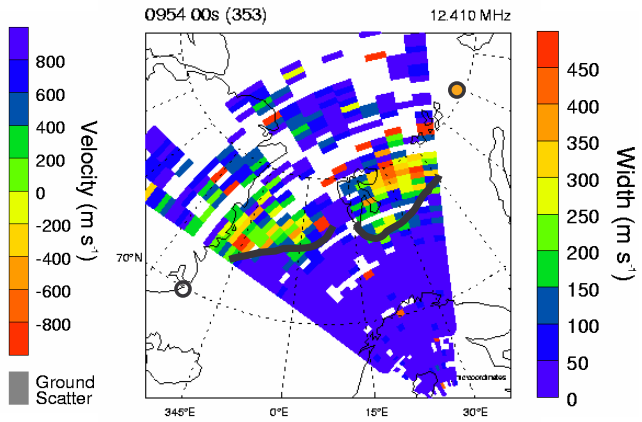

Ny-Ålesund $630.0 \mathrm{~nm}$

g

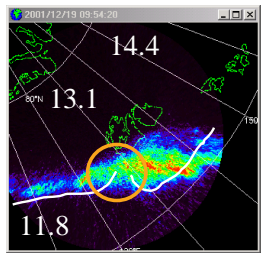

$220 \mathrm{~km}$

0954:20 UT

Fig. 11. Comparison of SuperDARN and optical data for the 2-min interval starting at 09:54 UT.

flow. These directions are expected poleward of the openclosed boundary, but not equatorward of it. The conclusion would be that the derived boundary is probably poleward of its actual location. Chisham et al. (2002a, b) suggested that a poleward displacement of the spectral width boundary should be expected when merging proceeds in the opposite hemisphere. A longer transmission time results for energy to traverse to the observational site and heat the plasma, during which the field lines have been convected poleward and westward. Even subsolar merging would provide some poleward displacement compared to merging near the local hemisphere cusp. Only ground scatter is observed by the Pykkvibaer radar from the region over Ny-Ålesund. This is typical of the data on this day.

Scans beginning at 09:54 UT are given in Fig. 11. DMSP F13 entered the radar fields of view during this period. Its position at the start of the scan is marked by the small orangefilled circle. The approximate position where DMSP later crossed the open-closed boundary is indicated by an open circle near the east coast of Greenland. The interval includes the onset of $557.7 \mathrm{~nm}$ emissions from west of Ny-Ålesund, which we identified with the IMF clock angles' switch to $\sim 180^{\circ}$. Six hundred and thirty-nm emissions are also more intense in this region. A comparison of images of Figs. 11e and 11f show that 557.7-nm emissions intensified during the sweep. Note that plasma flow recorded by Pykkvibaer near the west spectral-width boundary has switched back to strongly westward (blue), as it was at and prior to 09:52 UT. Plasma flows away from Hankasalmi remained very strong in the western beams, consistent with the spatial distribution observed by the DMSP F13 drift meter. The 557.7- nm emissions continued to be strongest near the spectral width boundaries. Again, the spectral width boundaries and the Hankasalmi flows are split in the region highlighted by the large orange circle.

Our comparison of radar and optical measurements at these and other times confirms the validity of using SuperDARN spectral-width boundaries as proxies for the openclosed field line boundary. It coincides with the location of the 557.7-nm emissions which we are associating with active merging. For the most part, it is also near the equatorward edge of the 630.0-nm emissions, at least in the eastern segment of the boundary. While the emissions just to the west of Ny-Ålesund came from locations close to the boundary, those coming from still further to the west tended to be poleward of the spectral-width boundary. The western segment of the spectral-width boundary was poleward of the eastern segment, especially when the clock angle was smaller, emphasizing that the source region for each is different, probably with longer transit times to the western source (Chisham et al., 2002). Note that the latitude difference between the western and eastern segments is less in Fig. 11, which represents the interval when the clock angle was near $180^{\circ}$. That the source regions differ is consistent with both the gap or 

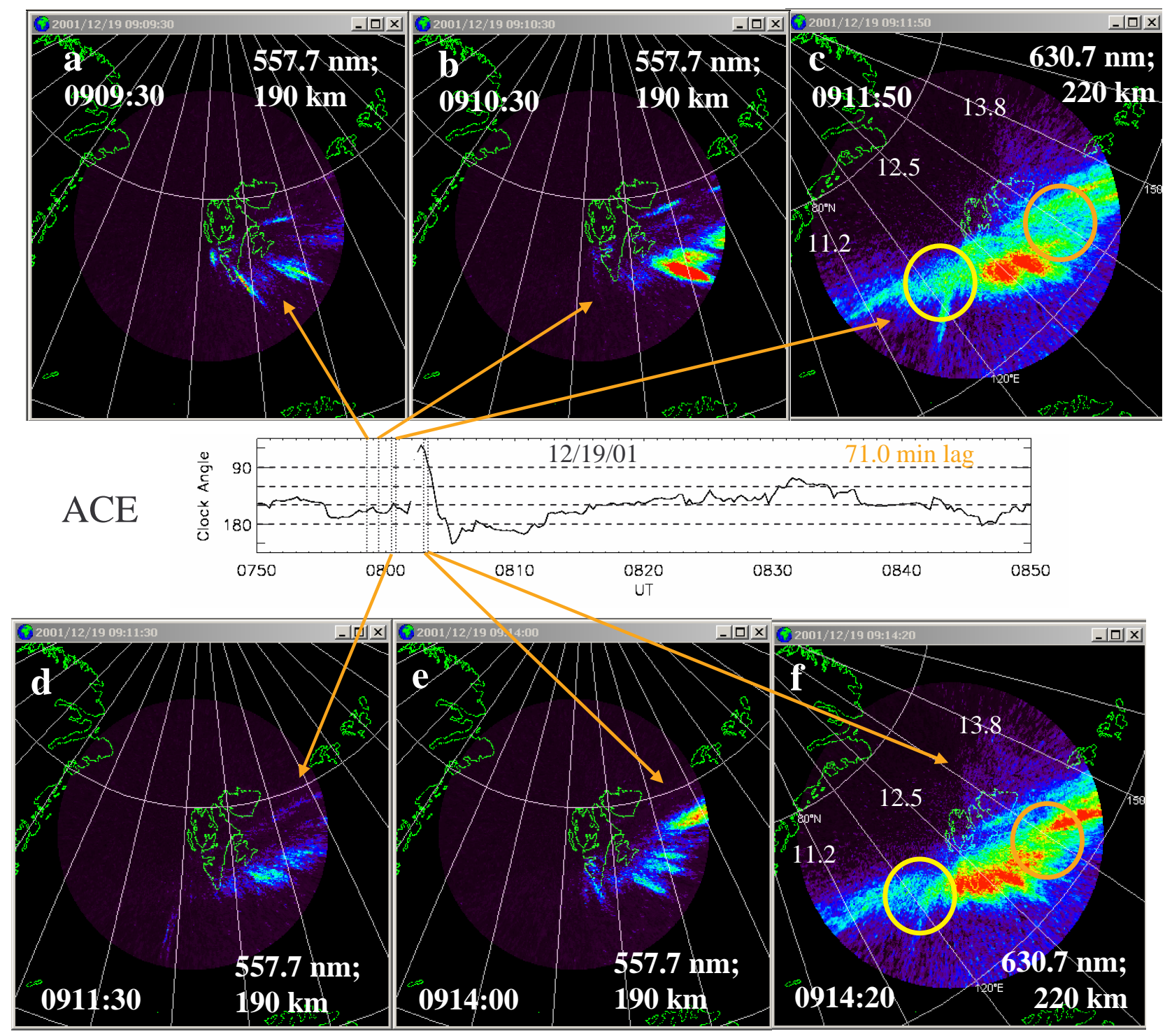

Fig. 12. All-sky images for the interval between 09:08 and 09:15 UT. The images are related to the appropriate ACE clock angle by the orange arrows, using a 71-min lag. (a), (b), (d), and (e) present 557.7-nm emissions. (c) and (f) present the nearest 630.0-nm images to the times of (d) and (e). The orange circles in (c) and (f) highlight a bifurcation in the emissions and are located at the same MLT as the orange circles in the previous figures. The yellow circles indicate a new change in character of the 630.0 nm emissions that is not visible in the all-sky field of view at the later times presented in previous figures.

bifurcation of 630.0-nm emissions and with the plasma velocity measurements just west of Svalbard, as seen by the Hankasalmi radar (compare Figs. 8-11).

\subsection{Earlier time intervals}

If this association of the 557.7-nm emissions with the IMF is robust, we should be able to associate other time intervals in the same way. A 15-min interval, 25 min after local magnetic noon, is explored in Fig. 12. This 09:10 to 09:15 UT period is one of enhanced emissions in both 557.7 and $630.0 \mathrm{~nm}$ as seen in the meridian scan data of Fig. 3. It was found that a longer lag of $71 \mathrm{~min}$ was needed, consistent with the variable lag displayed in Fig. 1. The 557.7-nm images for four times are displayed in Figs. 12a, 12b, 12d, and $12 \mathrm{e}$. Figures $12 \mathrm{c}$ and $12 \mathrm{f}$ display the closest $630.0-\mathrm{nm}$ image to those in Figs. 12d and 12e. The ACE clock angle was near $160^{\circ}$ until it abruptly shifted to less than $90^{\circ}$. Note that while Cluster also saw a decrease in $B_{Z}$, it did not reverse, indicating that the clock angle stayed greater than $90^{\circ}$ (Fig. 1d). Figures 12e and 12f correspond to the change in clock angle. The emission pattern in Fig. 12e shifted eastward relative to that found in the previous 557.7-nm images, as anticipated from the smaller clock angle. Figures $12 \mathrm{c}$ and $12 \mathrm{f}$ show strong emissions in the center of the band. The region to the east is also enhanced in Fig. 12f. The orange circle in each highlights a break in the emissions. This is located at the same local time, $\sim 13: 00$ MLT, as the break 
in the emissions (Figs. 8a-8c and 9a-9c) and in the spectral width boundary seen later near 09:50 UT. There is a second place where the character of the $630.0 \mathrm{~nm}$ emissions change, which is just before magnetic noon and highlighted in Fig. 12 by the yellow circles. This region would be out of the optical field of view in Figs. 8d-8f. The F13 DMSP potentials measured later in UT suggest that the tip of the morning convection cell should be associated with the emissions to the west of this second break. The strong emissions in between the yellow and orange circles appear at a time when the clock angle is of the order of $160^{\circ}$. Of importance here is the observation that the 557.7-nm emission locations change with clock angle and are continually varying with time and location, similar to the behavior observed near 09:50 UT, and to that anticipated from the conceptual picture of source regions of Maynard et al. (2001c). We return to these points in our discussion of possible source configurations.

4.8 Polar observations of $\mathrm{keV}$ electrons associated with merging

The previous sections established a plausible dependence of 557.7-nm variability on changes in the IMF clock angle. We next develop a rationale for interpreting these variations as signatures of merging based on Polar observations of enhanced electron fluxes conjugate to active merging sites on the magnetopause. Unfortunately, Polar's orbital plane is far from the dayside magnetopause and active merging sites in December, when optical images of cusp dynamics may be acquired at Ny-Ålesund. This association must be made indirectly. We begin by noting that precipitating electrons with energies (E) of $\sim 500 \mathrm{eV}$ stop at altitudes near $190 \mathrm{~km}$ (Rees, 1963). Higher energy electrons penetrate deeper; $1 \mathrm{keV}$ electrons lose most of their energy near $150 \mathrm{~km}$. Excitation of the 557.7-nm atomic oxygen line falls off at higher altitudes because of decreased neutral density. Significant 557.7-nm emissions, therefore, require electron fluxes with $E \geq 0.5 \mathrm{keV}$.

Magnetic merging at the magnetopause breaks electron gyrotropy and changes the pitch angle distribution of trapped plasma sheet electrons, creating a field-aligned high-energy tail in the distribution at energies $>0.5 \mathrm{keV}$. Maynard et al. (2003b) identified a number of merging events during Polar crossings of the dayside magnetopause, from signatures of accelerated ions, wave Poynting flux and, in some cases, successful Walén tests. In many cases merging occurred at high latitudes. Figure 13 presents data acquired during a sequence of magnetopause crossings on 1 April 2001. Figure 14a shows the field-aligned component of wave Poynting flux $\left(\Delta \mathbf{E} \times \Delta \mathbf{B} / \mu_{\mathbf{0}}\right)$, consistent with Alfvén waves carrying electromagnetic energy and field-aligned current away from merging sites (Maynard et al., 2003b). Figures 13b, $13 \mathrm{c}$, and $13 \mathrm{~d}$ show the total electron number density, the integral number density above $300 \mathrm{eV}$ and the integral electron number density above $1 \mathrm{keV}$, respectively. Figure $13 \mathrm{e}$ shows the $Z$ component of measured magnetic fields. In this case magnetopause crossings are identified by transitions from northward (red) to southward (blue) magnetic polarity.

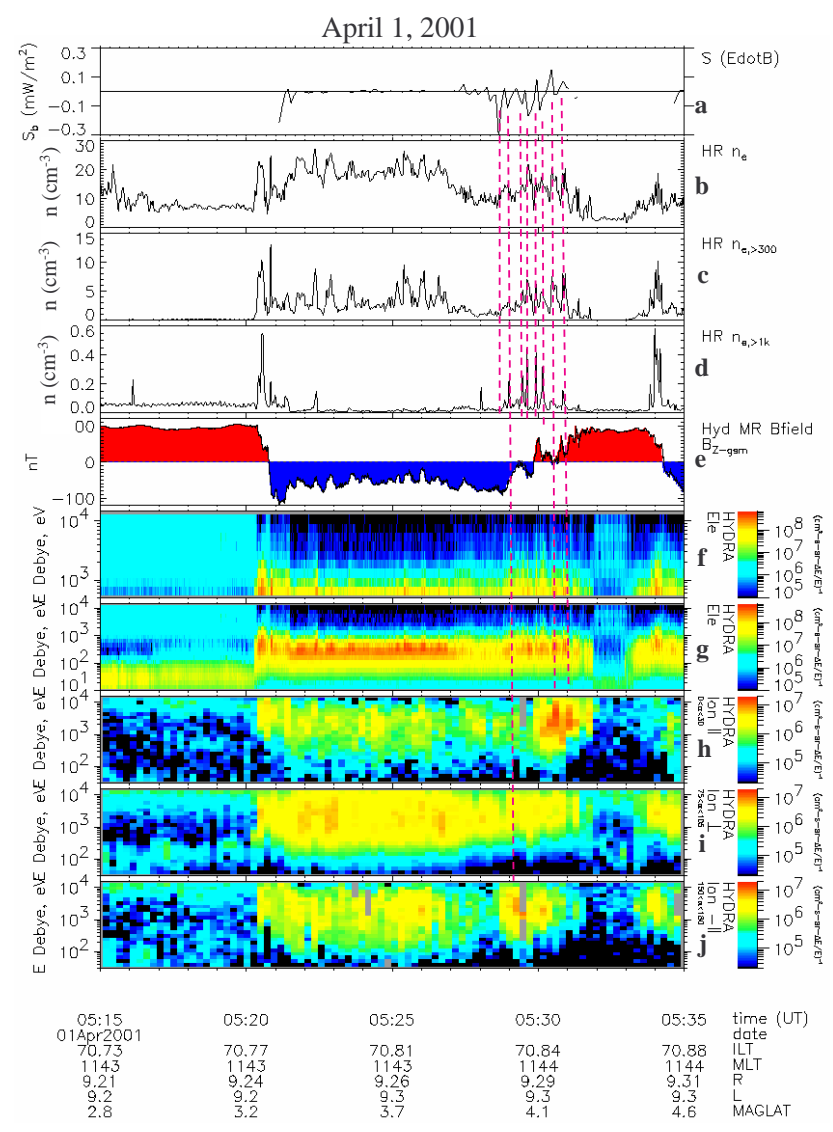

Fig. 13. Polar data acquired during three magnetopause crossings on 1 April 2001. From top to bottom the panels show (a) parallel Poynting flux, (b) electron number density, (c) number density of electrons with energies $>300 \mathrm{eV}$, (d) electron number density with energies above $1 \mathrm{keV}$, (e) northward component of the magnetic field measured at Polar, (f) and (g) spectrograms showing electrons with energies $>500 \mathrm{eV}$ and the fluxes over the whole instrument range, (h), (i), and (j) ion spectrograms showing the parallel, perpendicular and antiparallel fluxes. Accelerated ions, parallel Poynting flux and a high-energy tail on the electron distributions are seen on both sides of the magnetic field reversal in the central crossing. $\mathrm{KeV}$ electrons are seen on the magnetosphere side, especially at the other two magnetopause crossings. Poynting flux is not calculated because of the lack of knowledge concerning the correct background level for $B$ in the presence of the field reversal. Vertical lines highlight the correlation between the electron peaks, Poynting flux and accelerated ions.

The bottom half of Fig. 13 shows differential fluxes of electrons (Figs. 15f, 15g) and ions parallel (Fig. 13h), perpendicular (Fig. 13i) and antiparallel (Fig. 13j) to the magnetic field, measured by HYDRA and presented in energyversus-time spectrogram formats. Attention is directed to detections of an enhanced high-energy tail in electron fluxes, which are coincident with accelerated ions and wave Poynting flux during each magnetopause crossing. In particular, enhanced parallel ion fluxes (Fig. 13h) and wave Poynting vectors (Fig. 13a) propagated from latitudes below the spacecraft near 05:30 UT, corresponding to a clock angle near 


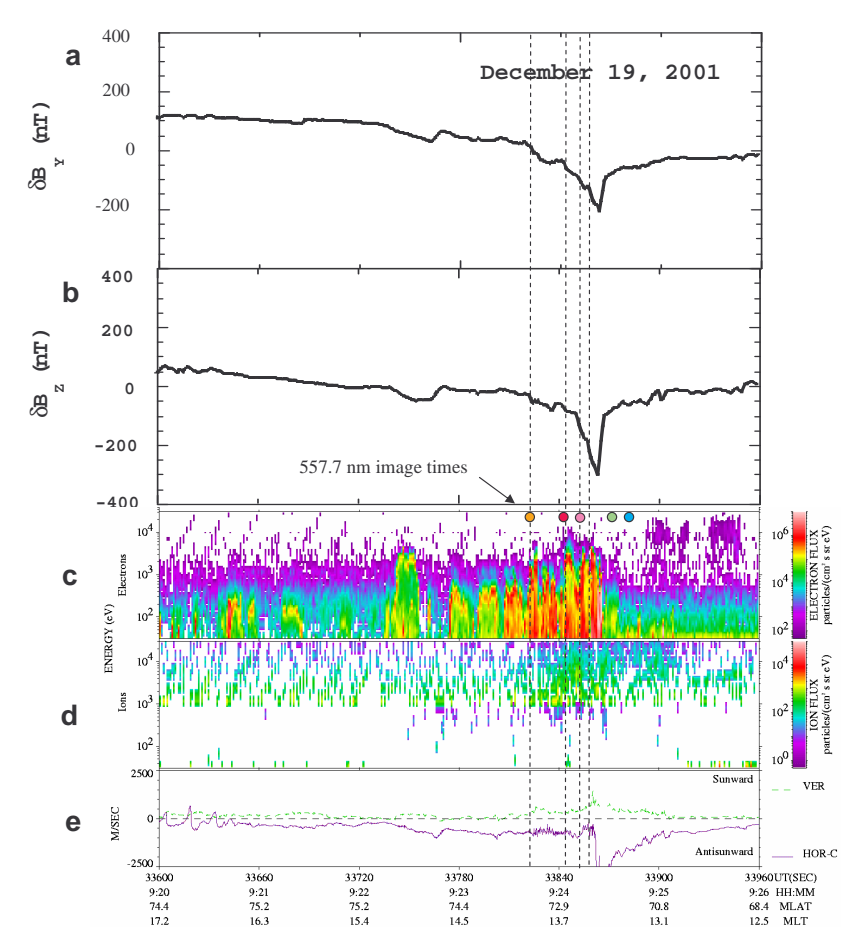

Fig. 14. High resolution DMSP F15 data showing two components of the perturbation magnetic field, electron and ion spectrograms, and the vertical and horizontal drifts. The negative slope of the perturbation magnetic fields indicates upward Region 1 currents. The colored circles denote the central times of 557.7-nm images presented in Fig. 15. Note that the first three correspond to electron enhancements, as well as intensification in the field-aligned currents (steeper slope) from the magnetometer records denoted by the vertical dashed lines.

$160^{\circ}$ (Maynard et al., 2003b, Table 1). The high-energy tails of electron distributions (Figs. 13c, 13d, and 13f) are also structured. Vertical dashed lines show that each spike in the high-energy electrons lines up with parallel Poynting flux for this crossing. Similar enhancements of high energy electrons are seen at the other two crossings where principal parallel ion fluxes are from above the spacecraft and the clock angle is near $130^{\circ}$. Note that if enhanced high-energy fluxes had tracked the total number flux (Fig. 13b), this would have indicated electron heating or just an increase in density rather than a change in the distribution. However, the enhancements are strongest and deviate most significantly from the integral number flux near magnetopause crossings. While quasineutrality requires that the bulk of the electron populations must travel with the ions, electrons in the high-energy tail can move ahead and reach the ionosphere before the main population. We expect to see these electrons first near the openclosed boundary, at footprints of field lines recently opened (Scudder et al., 1984).

Polar measurements provide evidence that electrons with sufficient energies to excite 557.7-nm emissions are a normal consequence of active merging. The skimming nature of Polar's orbit favors interpreting the enhancements observed in the high-energy electron fluxes as reflecting temporal varia- tions in merging activity rather than spatial structure. Since the 557.7-nm emission time constant is $\sim 1 \mathrm{~s}$, temporal variations in emissions should reflect temporal variations in the source. Away from active merging sites fluxes of electrons with $E>0.5 \mathrm{keV}$ is diminished. These facts support our use of 557.7-nm intensifications as proxies for active merging. To test this association, we return to the F15 measurements above Ny-Ålesund.

\subsection{DMSP electron observations associated with 557.7-nm emissions}

DMSP F15 passed close to the afternoon open-closed boundary to the southeast of Ny-Ålesund. Near 09:24 UT it encountered precipitating electrons in the vicinity of the observed optical emissions. Figure 14 shows an expanded plot of F15 measurements from this interval. Figures 14a and $14 \mathrm{~b}$ show $\delta B$ variations in the spacecraft-centered $Y$ and $Z$ coordinates (see Sect. 2). Between 09:24:00 and 09:24:24 $\Delta B_{Y}$ and $\Delta B_{Z}$ decreased from -38 and $-61 \mathrm{nT}$ to minima of -209 and $-301 \mathrm{nT}$, respectively. The region of negative slopes corresponds to a current out of the ionosphere, largely carried by precipitating electrons. The correlated changes in $\delta B_{Y}$ and $\delta B_{Z}$ of 171 and $240 \mathrm{nT}$ indicate that F15 crossed a FAC sheet at an attack angle of about $55^{\circ}$. The total magnetic perturbation in the sheet was $295 \mathrm{nT}$, which corresponds to $\boldsymbol{J}_{\|}$of $\sim 0.23 \mathrm{~A} / \mathrm{m}$. The average current density out of the ionosphere was about $1.6 \mu \mathrm{A} / \mathrm{m}^{2}$. In crossing the sheet the average integrated number flux of down coming electrons with energies between $30 \mathrm{eV}$ and $30 \mathrm{keV}$ was $\sim 10^{9} / \mathrm{cm}^{2}$-s-sr. Assuming a moderately field-aligned pitch angle distribution, the electron flux measured by $\mathrm{J} 4$ on F15 accounts for the $1.6 \mu \mathrm{A} / \mathrm{m}^{2}$ current density inferred from SSM measurements. Vertical dashed lines highlight parallel current maxima and tie these enhancements to the increases in the electron fluxes.

Color-coded dots shown at the top of the electron spectrogram denote the center times of the 5-s-interval of each 557.7-nm images shown in Fig. 15. The positions of F15 mapped along the magnetic field to $190 \mathrm{~km}$ are indicated by the color-coded dots. The 557.7-nm images have been mapped to $190 \mathrm{~km}$ as before. To better illustrate correspondence between 557.7-nm emissions and precipitating electrons the range of all-sky images were extended from zenith angles of $75^{\circ}$ to $80^{\circ}$. Comparison of Figs. 14 and $15 \mathrm{c}-15 \mathrm{e}$ reveal that the electron bursts observed between 09:24:00 and 09:24:30 UT match enhancements of 557.7-nm emissions. Some individual electron spectra in this region have "inverted V" signatures characteristic of field-aligned acceleration above the ionosphere. This acceleration is in addition to energies indicated by Polar as due to merging. We find that spectra outside of the "inverted V" structures have high-energy tail distributions similar to those observed by Polar when conjugate to merging sites on the magnetopause. Thus, the electron fluxes measured by DMSP appear to be the source of the 557.7-nm emissions. 


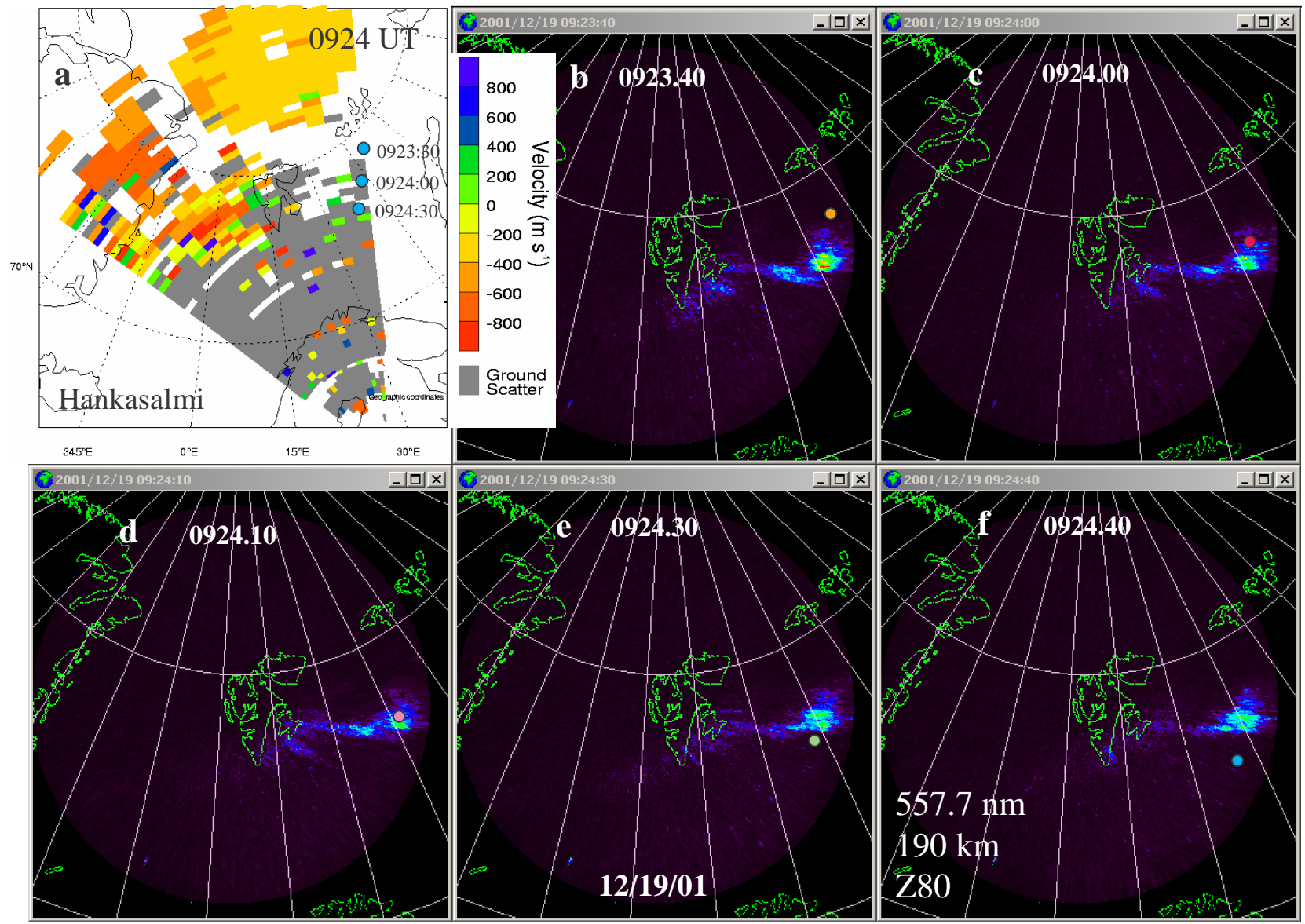

Fig. 15. (b - f) 557.7-nm all-sky images. The images have been extended to $80^{\circ}$ zenith angle to cover the mapped positions of DMSP (color-coded circles). Note that in both (c) and (d) the mapped position of DMSP coincides with the optical emissions. SuperDARN lineof-sight velocities from Hankasalmi are displayed in (a), along with the DMSP positions for three times. The large horizontal drift observed by DMSP near 09:24:30 UT is in a region apparently dominated by ground scatter observations.

Plasma velocities measured by the SuperDARN radar at Hankasalmi are shown in Fig. 15a for the interval starting at 09:24 UT. Mapped positions of DMSP at three times are overlaid on the velocity data. No significant velocities were detected by SuperDARN near the mapped DMSP locations. A strong burst of antisunward flow was observed by DMSP F15 co-located with the equatorward edge of these enhanced electron fluxes (Fig. 14e). A comparison of data in Figs. 14e with $14 \mathrm{a}$ and $14 \mathrm{~b}$ shows that the large flow region is primarily in the downward current region. We also checked the RPA measurements of the in-track component of plasma drift. Significant along-track flows are seen. However, their magnitudes and variability are not trustworthy because of the effects of small changes in the spacecraft potential on the analysis of the RPA current-voltage sweeps. The antisunward flows perpendicular to the orbit track, combined with probable flow along the orbit track, suggest that the resulting plasma velocity vector was nearly at quadrature to the SuperDARN line-of-sight. This may explain why the radar did not observe flows in that region. A second, and more probable, possibility is that real signals were obscured by ground scat- ter in this region. Finally, we note that similar large amplitude electric fields were observed by DE-2 at the open-closed boundary (Maynard, 1985; Maynard et al., 1991), and strong westward ion drifts in the cusp are associated with merging when $B_{Y}$ is positive (Maynard et al., 2003a).

\section{Discussion}

We have shown that temporal and spatial variations in all-sky images of 557.7-nm emissions are related to variations in the IMF clock angle. The spatial position is qualitatively consistent with the conceptual diagram of Maynard et al. (2001c), in which merging occurs around the rim of the high-latitude cusp at positions dependent on the IMF clock angle. We related the 557.7-nm emissions to a historical picture of the cusp based on 630.0-nm emission morphology, as well as SuperDARN and DMSP observations. Polar measurements show that high-energy electrons $(\geq 0.5 \mathrm{keV})$ are emitted from active merging sites. This observation provides a plausible source for electrons responsible for the green line emissions 
$\mathbf{a}$

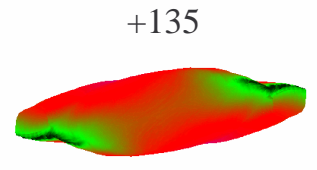

$0^{\circ}$ GSE Look angle

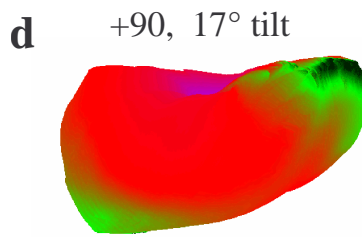

$0^{\circ}$ GSE Look angle b

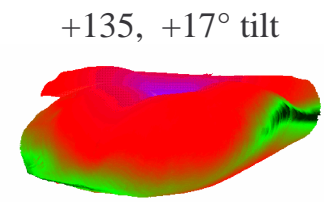

$0^{\circ}$ GSE Look angle

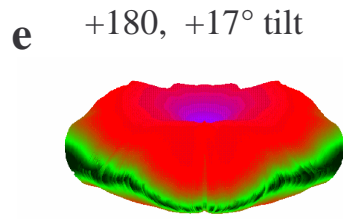

$0^{\circ}$ GSE Look angle c $-\mathrm{B}_{\mathrm{X}},+135$, no tilt

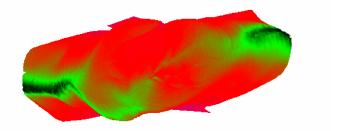

$0^{\circ}$ GSE Look angle

f
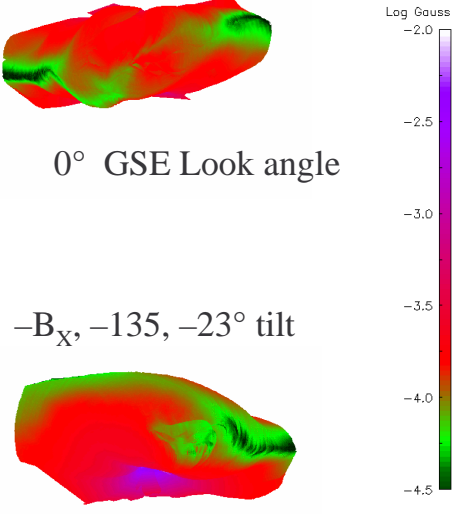

$0^{\circ}$ GSE Look angle

Fig. 16. Simulation results showing the magnetopause surface generated by the last closed field lines and colored with the log of the magnetic field magnitude. The low magnetic field values indicated by green or black serve as a proxy for possible merging locations. Various IMF clock angles, dipole tilts, and $B_{X}$ combinations are displayed in the six panels and discussed in the text.

and corroborates a suggestion of Maynard $(2003,2004)$ that the 557.7-nm emissions provide "television" pictures of the merging process on the dayside magnetopause. Before using this conclusion to infer temporal and spatial properties of dayside merging, we first examine the large-scale context of the solar wind/magnetosphere interaction provided by MHD modeling.

\subsection{IMF and dipole tilt control of merging locations}

MHD simulations provide a useful framework for interpreting data from multiple sources and locations (e.g. Maynard et al., 2001a, 2001b, 2003a, 2003b). The "sash", a low magnetic field region draped on the magnetopause which generally follows the anti-parallel merging locations of Crooker (1979) and Luhmann et al. (1984), was first identified in the MHD simulations (White et al., 1998 and then observed in a multi-satellite study by Maynard et al. (2001a). The position of the sash varies with clock angle (Siscoe et al., 2001) and extends around the dayside at the equator for a clock angle of $180^{\circ}$ and no dipole tilt. Maynard et al. (2003b) showed that even for a $180^{\circ}$ clock angle, dipole tilts push merging sites in the noon meridian away from the equator toward higher latitudes in the hemisphere tilting away from the Sun. Russell et al. (2003) have looked for possible antiparallel merging locations on the magnetopause in MHD simulations to show that dipole tilt greatly influences the location and length of the merging separator. We use the existence of low magnetic fields on the magnetopause, found in our simulations, as a proxy for identifying locations of potential merging sites.

Figure 16 presents results from a sequence of MHD simulations using the ISM model (White et al., 2001). Each panel shows the three-dimensional magnetopause formed by the last closed field lines and colored by the log of the magnetic field strength. The view is from the Sun. Green and black represent weak magnetic fields. Figure 16a shows the magnetopause for no dipole tilt and a clock angle of $135^{\circ}$, the prevalent clock angle for data used in the present study. Note that the green regions signifying low magnetic field strengths are at high latitudes in opposite hemispheres and are clearly split. In Fig. $16 \mathrm{~b}$ a $17^{\circ}$ forward dipole tilt is added. The green region now extends along the dayside on the front rim of the cusp in the Southern Hemisphere. The Northern Hemisphere merging site moves to mid latitudes on the flank. The simulation in Fig. 16c has no dipole tilt, but a negative IMF $B_{X}$ has been added. Crooker (1992), in a study of effects on lobe cell merging, first recognized the complementarity of both dipole tilt and $B_{X}$, concluding that they have comparable effects on merging. The results appear similar to Fig. $16 \mathrm{~b}$ where the low field region extends toward noon along the front rim of the Southern Hemisphere cusp. In this case the merging region is intermittent and located near the site of the first contact with the IMF. This has an opposite effect to the dipole tilt seen in Fig. 16b. Figures 16d and 16e complete the $17^{\circ}$ variation with clock angle for the $90^{\circ}$ and $180^{\circ}$ cases. Figure $16 \mathrm{~d}$ shows a clear bifurcation between the Northern and Southern Hemisphere merging sites for pure $B_{Y}$.

Figure 16e generalizes the result of Maynard et al. (2003b) at the noon meridian to show that the merging site moves to southern high latitudes all along the dayside. Figure $16 \mathrm{f}$ simulates a winter solstice situation with $-B_{X}$, except that the clock angle is $-135^{\circ}$ to place the Northern Hemisphere merging site on the dawn side of noon. The green region extends across noon at northern high latitudes and the Southern Hemisphere green region moves closer to the equator. Attention is directed to a patch of green close to the dusk side of the nose. This is a time-dependent feature similar in nature to 
$-135^{\circ}$ Clock angle $\&-B_{X}-23^{\circ}$ tilt

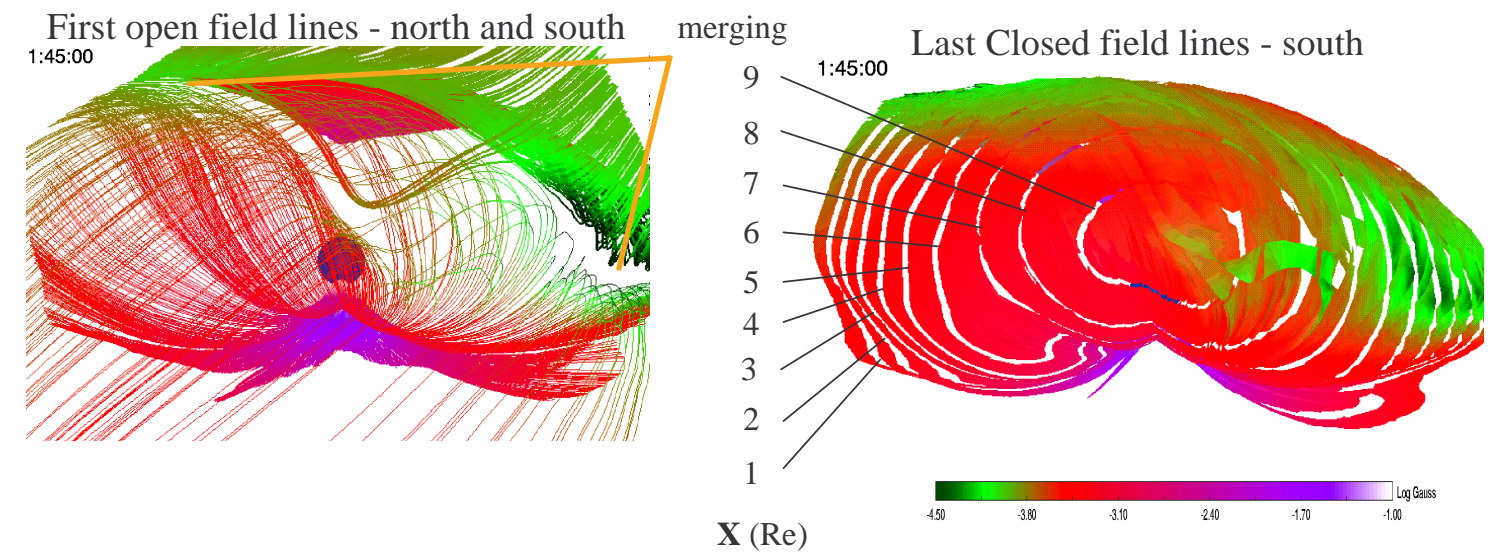

Fig. 17. The right panel expands Fig. $16 \mathrm{f}$ and builds the magnetopause in $1 \mathrm{R}_{E}$ segments to show the 3 -D aspects of the surface with a negative $135^{\circ}$ clock angle, negative $B_{X}$ and a $-23^{\circ}$ dipole tilt, characteristic of winter and similar to the event discussed (note that the clock angle is negative so that post-noon features in the data would be seen pre-noon in the simulation). The left plot traces the first open field lines from both Northern and Southern Hemisphere cusps. Open field lines from opposite hemispheres meet over the green areas in the right panel, which we identify as proxies for merging locations. The dip near noon is different from all other Northern Hemisphere open field lines and matches Southern Hemisphere lines that meet. This is a transient event in the simulation.

the feature in Fig. 16c located near the point of first contact with the IMF.

Figure 17 is a closer look at the simulation of Fig. 16f. In the right panel the closed surface has been built in $1 \mathrm{R}_{E}$ segments to improve the 3-D perspective in this head-on view. The gaps mark integer values of $X$. The left panel shows the first open field lines traced from both hemispheres. The orange lines point to the principal merging locations where the field lines diverge. The field lines traced from the Southern Hemisphere extend to high latitudes in the north and meet those from the Northern Hemisphere in the green region shown in the right panel. Note that a few field lines from both hemispheres meet near the nose in the temporally varying green patch. The size of this patch is $\sim 1 \mathrm{R}_{E}$, and associated field lines follow paths completely different from their neighbors. In comparing this with experimental results presented above, recall that the prenoon region of the simulation must be compared to the postnoon results in the observations.

Figures 16 and 17 clearly show high-latitude merging regions in both hemispheres on opposite sides of noon. For some clock angles and tilts, the merging region clearly bifurcates in local time as well as by hemisphere. As the clock angle and tilt change, the central region closer to noon may or may not develop new merging sites. Thus, MHD simulations identify three potential merging sites. Very large tilts not only cause the central merging site to move toward high latitudes, but also cause the side merging site in the hemisphere tilted toward the Sun to move closer to the equator. The presence of $B_{X}$ excites transient merging events in the central region, near where the phase plane first contacts the magnetopause. The simulations suggest that, except for these transient events, merging in the middle segment occurs at high latitudes near the front rim of the cusp in the hemisphere tilted away from the Sun.

\subsection{Spatial structure of the source regions}

The simple concept of cusp bifurcation (Maynard et al, 2001c; Coleman et al.; 2001; Chisham et al., 2002a) applies when $B_{Y}$ is the dominant component. Measurements shown in Fig. 12 suggest that there may be three source regions, and MHD simulations (Figs. 16 and 17) corroborate this conjecture. When the IMF clock angle is $\geq 135^{\circ}$, potential merging sites may develop along the rim of the cusp in the hemisphere tilted away from the Sun, dividing the cusp into three segments. This is apparent in comparing Fig. 12 with other all-sky images. The source bifurcation, marked by the orange circle in Fig. 12 and in Figs. 8 and 9, remains near 13:00 MLT for at least $30 \mathrm{~min}$. This also suggests that the shift in emissions to earlier MLTs as the clock angle increased (Figs. 7 and 9) located their source in the middle segment. Figure 12 also shows a second change in the character of prenoon emissions. In the middle region 557.7-nm emissions are variable, while the 630.0-nm emissions are strong. Figures $12 \mathrm{e}$ and $12 \mathrm{f}$ imply that the merging transitions to the side area, located post 13:00 MLT as the clock angle rapidly decreases. The post-13:00 MLT location is consistent with the findings of Maynard et al. (2003b) that merging was at high latitudes for clock angles $<150^{\circ}$. In their 12 March 2001 event with a clock angle of $\sim 135^{\circ}$, the merging location mapped close to 14:00 MLT. The $160^{\circ}$ clock angle associated with most of the data in Fig. 12 and the strong emissions suggest that merging may be following the low field locus at high latitudes indicated by the simulations. The lack of significant prenoon 557.7-nm emissions argues for an opposite 
hemisphere source for that segment of the cusp. Emissions near 13:00 MLT in Fig. 9a tend to extend across the gap, as do the $557.7 \mathrm{~nm}$ emissions in this situation, with a clock angle approaching $\sim 180^{\circ}$. Multiple source regions may be active when the tilted IMF phase plane impacts the magnetopause, especially when the IMF is changing. Due to the tilt of the phase plane, draping will take longer in the hemisphere away from the tilt, delaying merging in that hemisphere relative to the other. Because real conditions continually change, the character of the middle segment will routinely alter in time. Note that Figs. 16 and 17 suggest that the location of merging in the middle segment may often be at high latitudes near the front rim of the cusp when there is significant dipole tilt. The high-latitude location is significantly different from component merging separators which pass through the sub-solar region (e.g. Gonzales and Mozer, 1974). In addition, the simulations show that transient events may occur at a more sub-solar locale. Evidence for multiple sources for DMSP cusp particles has also been presented by Wing et al. (2001), who suggested that high-latitude and sub-solar merging regions can be active together.

\subsection{Implications relative to the source electrons}

We have used Polar measurements with signatures of merging identified by Maynard et al. (2003b) to suggest a plausible source for electrons that produce 557.7-nm emissions. Where parallel Poynting flux is enhanced and ion acceleration is observed, the electrons have a high-energy tail with significant fluxes $>500 \mathrm{eV}$ (Fig. 13). These electrons may run ahead of the bulk population to be first to travel down the magnetic field line to the ionosphere and create the emissions. As they emerge from the merging site along the separatrix, their ionospheric footprints should be close to the open-closed boundary. Figures 14 and 15 connect DMSP electron observations with the short-duration enhancements in 557.7-nm emissions. Filamentary electron structures were observed on the first sounding rocket into the cusp (Maynard and Johnstone, 1974). The observed electron bursts had significant fluxes above $500 \mathrm{eV}$. Liouville's theorem was used to show that the phase space densities were similar to those in the magnetosheath. Maynard and Johnstone postulated that small regions of direct access were imbedded within the larger cusp region. Their observations have similarities to the structured precipitation reported here. In addition, Moen et al. (2001b) hypothesized that reconnection was associated with an observation of $1-2 \mathrm{keV}$ cusp electrons, measured by Cluster, and with Type 7 aurora (Sandholt et al., 1998) in the post-16:00 MLT sector.

Some of these electrons appear to be accelerated above the energies observed by Polar with the characteristic signature of acceleration from a field-aligned potential above the ionosphere. Alfvén waves carry field-aligned currents along the separatrix, communicating the new magnetic field configuration at the magnetopause. Just as in the upward current region of the nightside aurora, field-aligned potentials and electron acceleration develop when the immediate source of electrons in the loss cone is inadequate to carry the required field-aligned current (Lyons, 1981).

A second aspect of particle acceleration at the merging site is that when merging occurs at high latitudes, the flow in the magnetosheath away from the stagnation point at the nose influences the energy of the particles in the two exhaust regions. Just as ions may be decelerated rather than accelerated by merging in the upstream exhaust (Scudder, 1984; Aggson et al., 1984), we may expect electrons that propagate away from the separator to the near (far) ionosphere to be at higher (lower) energies. During this whole sequence, no 557.7-nm emissions were seen in the prenoon segment of the cusp, a region which we have suggested is tied to a Southern Hemisphere source. This contrasts with prenoon observations of temporally and spatially dependent $557.7-\mathrm{nm}$ emissions when $B_{Y}$ is negative (Maynard, 2003b), which are analogous to postnoon observations presented here when $B_{Y}$ is positive. Electrons coming from the upstream side of the separator are expected to have less energy and may not produce optical signatures when they finally make it to the opposite hemisphere. Emissions should be strongest when the source electrons are produced by merging near the front rim of the cusp in the local hemisphere.

\subsection{Relating SuperDARN spectral-width boundaries to merging locations}

Chisham et al. (2002a, b) used the latitudinal difference in local time of spectral-width boundaries as indicators of cusp bifurcation caused by merging in opposite hemispheres. Merging products from the local hemisphere reach the ionosphere more quickly than those from the opposite hemisphere. Convection displaces the parts of the boundary controlled from the opposite hemisphere poleward, or in this case, toward dawn as well. Thus, the spectral-width boundary ceases to indicate accurately the open-closed boundary which may shift significantly poleward depending on the convection speed. Subsolar merging causes a similar but less pronounced poleward displacement of the spectral width boundary. Strong velocities toward Pykkvibaer in Fig. 11 (highlighted by the red square), which straddle the western boundary segment, must be located poleward of the openclosed boundary and driven from the Southern Hemisphere. The plasma velocities observed by the Hankasalmi radar are poleward of those seen from Pykkvibaer. Hence, the combined vector is directed antisunward and toward dawn, a direction expected from convection patterns located poleward of the convection reversal (e.g. Weimer, 2000). Boundaries shown in Figs. 10 and 11 and at other times analyzed, but not shown, indicate significant differences in latitude of the two spectral-width boundary segments, consistent with opposite hemisphere merging (Chisham et al., 2002a, b). This pre-noon region has no 557.7-nm emissions.

Near Ny-Ålesund we see 557.7-nm emissions associated with the southward turning of the IMF. The western segment of the spectral-width boundary in Fig. 11 is close to the emission boundary, but is still poleward. The location is well 
post-noon. Maynard et al. (2003b) showed that dipole tilt displaces merging off the equator away from the tilt, even for pure southward IMF. This suggests that the optical response to the southward turning still places merging in the Northern Hemisphere, but perhaps equatorward of the cusp rim as with merging in the post-13:00 MLT segment (yellow and red fiducials in Figs. 4-7). The ISM simulation results (Fig. 17) show a poleward displacement of the merging region across noon.

Maynard et al. (2001b) found that because of tilted phase planes, the two hemispheres can respond to the same IMF signal at very different times. Weimer et al. (2002) found that the tilt of these phase planes could change significantly on scales of tens of minutes. In the present case, the dipole tilt causes a first impact to occur near a Southern Hemisphere merging site. A change in clock angle to $\sim 180^{\circ}$ at ACE appears to be related to Northern Hemisphere signatures with a 69.5-min lag at 09:56 UT. It is reasonable that the prenoon Southern Hemisphere merging site sees and responds to this change at an earlier time. We suggest that the brief change in velocity from toward to away near the western segment of the spectral width boundary seen at Pykkvibaer (highlighted by the red box in Fig. 10) represents a Southern Hemisphere response to that brief change in clock angle. Again, we observed no 557.7-nm optical responses from this region. The first indications of 557.7-nm signals related to the IMF turning which occured at 09:53:30 UT in Fig. 2, which is tied to earlier variations in the IMF with positive $B_{Y}$. The exact nature of the reaction in the Southern Hemisphere is not discernible from our data set.

\subsection{Temporal and spatial structure of merging}

When IMF clock angles are $\leq 150^{\circ}$ most 557.7-nm emissions appeared after 13:00 MLT and are probably associated with the subset of cusp aurora that Sandholt et al. (1998) identified as category 5 or 7 . We associate this with high-latitude merging in the local hemisphere. The location of these 557.7-nm emissions is consistent with the SuperDARN proxy for the open-closed field line boundary. Both the optical and SuperDARN data fit the spatial snapshot given by a concurrent DMSP pass. We identified a probable source of the 557.7-nm emissions as the high-energy tail of the electron distribution from electrons accelerated in the merging process on the magnetopause. These electrons are detected by Polar when active merging is present. In making this association, spatial and temporal variations, evident in Figs. 4 and 5 , constrain and clarify our picture of the variability of magnetopause merging.

First, merging can occur simultaneously at multiple locations and, in this data set, does not appear to be steady at any location. Preferred merging sites closely follow the IMF clock angles. Second, merging at particular locations occurs on time scales of $30 \mathrm{~s}$ to several minutes. That an individual site turns off does not mean that the overall merging process has completely stopped. Third, from 09:35 to 10:00 UT, 557.7-nm emissions never completely vanish. Flow enhance- ments may be associated with each region when it activates, as observed by Maynard et al. (2003b) in the SuperDARN and Cluster data, but the net flow is spread out in the large convection cell. Merging is an intermittent process consistent with expectations for regions where the tangential component of magnetosheath plasma velocity exceeds the Alfvén speed (Rodger et al., 2000; Scudder, 1984). These conclusions apply to high-latitude merging in the local hemisphere. The Southern Hemisphere merging site may be operating similarly but with different structures, and is perhaps responding to a different portion of the solar wind data stream. Note that Maynard et al. (2001c) detected localized optical responses to opposite hemisphere merging near the tip of the small convection cell represented in inertial coordinates.

Field lines, tied to the opposite-hemisphere merging sites, drape over the magnetopause and unwind as they are pulled tailward by the solar wind, forming an open boundary layer at the magnetopause (Maynard et al., 2001a). In this region the effects of multiple merging sites may be more concentrated in driving convection in the ionosphere from their draping. Fast flow in the throat region between the two cells is evident in the convection patterns (Heppner and Maynard, 1987). DMSP F13 data in Fig. 2 show the fast flow and cusp particles near 10:00 MLT, but the electrons poleward of the open-closed field line are lower in energy and do not produce fast-response $557.7 \mathrm{~nm}$ signals.

\section{Summary}

We conclude that magnetic merging at the dayside magnetopause is temporally varying on time scales of $30 \mathrm{~s}$ to $3 \mathrm{~min}$ and occurs asynchronously at multiple sites simultaneously. These conclusions are based on the variability of 557.7-nm emissions in high-temporal resolution all-sky images.

To establish the validity of interpreting all-sky images of ionospheric 557.7-nm emissions as "television" pictures of merging events at the magnetopause (Maynard, 2003, 2004), we have analyzed images acquired on 19 December 2001, at 10 -s intervals at $\mathrm{Ny}-\AA$ Ålesund. Characteristic variations in the optical measurements coincided with times when significant variations in the IMF clock angle should reach the magnetosphere, based on the variable lag time analysis (Weimer et al., 2002) of ACE and Cluster data. Responses in the highresolution images also correlate with spectral-width boundaries and velocities measured by two SuperDARN radars.

A plausible mechanism for the source of the emissions is the high-energy tail of the electrons energized at the merging site. Polar observations at the magnetopause during merging events provide direct evidence (albeit at other times) that links enhanced high-energy tails of the electrons with merging. A DMSP F15 overflight measured the actual electron fluxes causing the emissions. In some cases, these fluxes exhibited "inverted- $V$ " structures, indicating the probable occurrence of additional acceleration above the ionosphere. Magnetometer measurements placed these fluxes in regions of upward field-aligned currents. 
Comparisons of optical and SuperDARN data suggest that the cusp divides into three source regions for positive IMF $B_{Y}$ conditions. Emissions were located east of 13:00 MLT when clock angles were $\sim 150^{\circ}$. For larger clock angles emissions were seen between noon and 13:00 MLT. No significant 557.7-nm emissions were detected in the prenoon region. MHD simulations indicate that the source for the post 13:00 MLT emission is near the rim of the high-latitude Northern Hemisphere cusp. The prenoon source region is tied to the Southern Hemisphere merging site. Simulations also suggest that the source of the emissions between 12:00 and 13:00 MLT for very large clock angles is likely to be near the front rim of the Northern Hemisphere cusp. For a positive dipole tilt merging moves off the equator, away from the tilt, to higher magnetic latitudes in the Southern Hemisphere, even for IMF clock angles of $180^{\circ}$ (Maynard et al., 2003b). For winter solstice tilts it moves to the front rim of the Northern Hemisphere (Fig. 19). Furthermore, simulations show transient effects in the noon region, where time-dependent merging may occur near sites of the first contacts of interplanetary electric field phase planes with the magnetopause and at lower latitudes in the opposite hemisphere.

Our results establish that 557.7-nm images represent new tools for investigating the spatial and temporal evolution of the merging process. These images constrain possible interpretations of the locations and rates of the process. Future studies of other events using two-dimensional pictures afforded by such images promise to reveal more about the complex coupling of the solar wind to the magnetosphereionosphere system.

Acknowledgements. Special thanks are due to A. Egeland, whose efforts over two decades have facilitated the optical measurements at Svalbard, and to R. A. Greenwald for similar support in the establishment of the SuperDARN network. We thank B. Lybekk for the meridian scanning photometer data. We are grateful to $\mathrm{C}$. W. Smith and D. J. McComas for use of the ACE magnetic field and plasma data. We thank F. S. Mozer for use of the Polar electric field data. L. Gentile provided valuable assistance in editing the test. The work at MRC was supported by contracts NASW-01011 and NASW-02017, NASA grant NAG5-3182 (subcontract to the University of California, Berkeley) and by the NASA Sun-Earth Connections Theory Program Grant NAG5-8135 (subcontract to Boston University). The ISM was developed under sponsorship of the Defense Threat Reduction Agency, 45045 Aviation Drive, Dulles, VA 20166-7517. We also acknowledge support from the Norwegian Research Council, NASA grants and contracts NAG5-2231, NAG5-3328, NAG5-7721, NAG5-7712, NAG57883 NAG5-8119, NAS5-30302, NAS5-30316 and NASW-99014, NAG5-10883, NAG5-121189, and NAG5-11803, and from AFOSR tasks 2311PL13 and 2311AS. The operations of the Hankasalmi and Pykkvibaer radars are funded by PPARC.

Topical Editor T. Pulkkinen thanks F. Søraas and another referee for their help in evaluating this paper.

\section{References}

Aggson, T. L., Maynard, N. C., Ogilvie, K. W., Scudder, J. D., and Gambardella, P. J.: Observation of plasma deceleration at a rotational magnetopause discontinuity, Geophys. Res. Lett., 11, 8-11, 1984.

Baker, K. B., Dudeney, J. R., Greenwald, R. A., Pinnock, M., Newell, P. T., Rodger, A. S., and Meng, C.-I.: HF-radar signatures of the cusp and the low- latitude boundary layer, J. Geophys. Res., 100, 7671, 1995.

Baker, K. B., Rodger, A. S., and Lu, G.: HF-radar observations of the dayside magnetic merging rate: A geospace environment modeling boundary layer campaign study, J. Geophys. Res., 102, 9603, 1997.

Balogh, A., Carr, C. M., Acuña, M. H., Dunlop, M. W., Beek, T. J., Brown, P., Fornaçon, K.-H., Georgescu, E., Glassmeier, K.H., Harris, J., Musmann, G., Oddy, T., and Schwingenschuh, K.: The Cluster magnetic field investigation: overview of in-flight performance and initial results, Ann. Geophys., 19, 1207-1217, 2001.

Chisham, G. and Freeman, M. P.: A technique for accurately determining the cusp-region polar cap boundary using SuperDARN HF radar measurements, Ann. Geophys., 21, 983, 2003.

Chisham, G., Pinnock, M., Coleman, I. J., Hairston, M. R., and Walker, A. D. M.: An unusual geometry of the ionospheric signature of the cusp: Implications for magnetopause merging sites, Ann. Geophys., 20, 29, 2002a.

Chisham, G., Coleman, I. J., Freeman, M. P., Pinnock, M., and Lester, M.: Ionospheric signatures of split reconnection $\mathrm{X}$-lines during conditions of IMF $B_{Z}<0$ and $\left|B_{Y}\right| \sim\left|B_{Z}\right|$ : Evidence for the antiparallel merging hypothesis, J. Geophys. Res., 107(A10) 1323, doi:10.1029/2001JA009124, 2002b.

Coleman, I. J., Chisham, G., Pinnock, M., and Freeman, M. P.: An ionospheric convection signature of antiparallel reconnection, J. Geophys. Res., 106, 28 995, 2001.

Crooker, N. U.: Dayside merging and cusp geometry, J. Geophys. Res., 84, 951, 1979.

Crooker, N. U.: Reverse convection, J. Geophys. Res., 97, 19363 , 1992.

Fasel, G. J.: Dayside poleward moving auroral forms: A statistical study, J. Geophys. Res., 11 891-11 905, 1995.

Greenwald, R. A., Baker, K. B., Dudeney, J. R., Pinnock, M., Jones, T. B., Thomas, E. C., Villain, J.-P., Cerisier, J.-C., Senior, C., Hanuise, C., Hunsucker, R. D., Sofko, G., Koehler, J., Nielsen, E., Pellinen, R., Walker, A. D. M., Sato, N., and Yamagishi, H.: DARN and SuperDARN: A global view of high latitude convection, Space Sci. Rev., 71, 761-796, 1995.

Gonzalez, W. D. and Mozer, F. S.: A quantitative model for the potential resulting from reconnection with arbitrary interplanetary magnetic field, J. Geophys. Res., 79, 4186, 1974.

Hain, K.: The partial donor cell method, J. Comput. Phys., 73, 131, 1987.

Hardy, D. A., Schmidt, K. L., Gussenhoven, M. S., Marshall, F. J., Yeh, H. C., Shumaker, T. L., Huber, A., and Pantazis, J.: Precipitating electron and ion detectors (SSJ/4) for the block 5D flights 4-10 DMSP satellites: Calibration and data presentation, Tech. Rep. AFGL-TR-84-0317, Air Force Geophys. Lab., Hanscom Air Force Base, Mass., 1984.

Harvey, P., Mozer, F. S., Pankow, D., Wygant, J., Maynard, N. C., Singer, H., Sullivan, W., Anderson, P. B., Pfaff, R., Aggson, T., Pedersen, A., Fälthammar, C.-G., and Tanskannen, P: The electric field instrument on the Polar satellite, Space Sci. Rev., 71, 
583-596, 1995.

Heppner, J. P. and Maynard, N. C.: Empirical High Latitude Electric Field Models, J. Geophys. Res., 92, 4467-4489, 1987.

Luhmann, J. G., Walker, R. J., Russell, C. T., Crooker, N. U., Spreiter, J. R., and Stahara, S.: Patterns of potential magnetic field merging sites on the dayside magnetopause, J. Geophys. Res., 89, 1739, 1984.

Lyons, L. R.: Discrete aurora as the direct result of an inferred highaltitude generating potential distribution, J. Geophys. Res., 86, $1-8,1981$

Maynard, N. C.: Structure in the DC and AC electric fields associated with the dayside cusp region, in: The polar cusp, edited by Holtet, J. A. and Egeland, A., 305-322, D. Reidel Publishing Co., 1985.

Maynard, N. C.: Svalbard: A window for understanding tempo$\mathrm{ral} / \mathrm{spatial}$ aspects of solar wind coupling to the magnetosphere and ionsphere, in: Egeland Symposium on Auroral and Atmospheric Research, edited by Moen, J. and Holtet, J. A., Department of Physics, University of Oslo, ISBN 82-91853-09-6, 7589, 2003.

Maynard, N. C.: Coupling the solar-wind/IMF to the ionosphere through the high latitude cusps, Surveys in Geophysics, in press, 2004

Maynard, N. C. and Johnstone, A. D.: High latitude dayside electric field and particle measurements, J. Geophys. Res., 79, 31113123, 1974.

Maynard, N. C., Aggson, T. L., Basinska, E. M., Burke, W. J., Craven, P., Peterson, W. K., Sugiura, M., and Weimer, D. R.: Magnetospheric boundary dynamics: DE-1 and DE-2 observations near the magnetopause and cusp, J. Geophys Res., 96, 3505-3522, 1991.

Maynard, N. C., Savin, S., Erickson, G. M., Kawano, H., Němeček, Z., Peterson, W. K., Šafránoková, J., Sandahl, I., Scudder, J. D., Siscoe, G. L., Sonnerup, B. U. Ö., Weimer, D. R., White, W. W., and Wilson, G. R.: : Observation of the magnetospheric "sash" and its implications relative to solar-wind/magnetosphere coupling: A multisatellite event analysis, J. Geophys. Res., 106, 6097-6122, 2001a.

Maynard, N. C., Siscoe, G. L., Sonnerup, B. U. Ö., White, W. W., Siebert, K. D., Weimer, D. R., Erickson, G. M., Schoendorf, J. A., Ober, D. M., and Wilson, G. R.: The response of ionospheric convection to changes in the IMF: Lessons from a MHD simulation, J. Geophys. Res., 106, 21 429-21 451, 2001 b.

Maynard, N. C., Burke, W. J., Sandholt, P. E., Moen, J., Ober, D. M., Lester, M., Weimer, D. R., and Egeland, A.: Observations of simultaneous effects of merging in both hemispheres, J. Geophys. Res., 106, 24 551, 2001c

Maynard, N. C., Burke, W. J., Moen, J., Ober, D. M., Scudder, J. D., Sigwarth, J. B., Siscoe, G. L., Sonnerup, B. U. Ö., White, W. W., Siebert, K. D., Weimer, D. R., Erickson, G. M., Frank, L. A., Lester, M., Peterson, W. K., Russell, C. T., Wilson, G. R., and Egeland, A.: Responses of the open-closed field line boundary in the evening sector to IMF changes: A source mechanism for Sun-aligned arcs, J. Geophys. Res., 108(A1), 1006, doi10.129/2001JA000174, 2003a.

Maynard, N. C., Ober, D. M., Burke, W. J., Scudder, J. D., Lester, M., Dunlop. M., Wild, J. A., Grocott, A., Farrugia, C. J., Lund, E. J., Russell, C. T., Weimer, D. R., Siebert, K. D., Balogh, A., Andre, M., and Rème, H.: Polar, Cluster and SuperDARN evidence for high latitude merging during southward IMF: temporal/spatial evolution, Ann. Geophys., 21, 2233-2258, 2003b.

McComas, D. J., Bame, S. J., Barber, P., Fieldman, W. C., Phillips,
J. L., and Riley, P.: Solar wind electron, proton, and alpha monitor (SWEPAM) on the advanced composition explorer, Space Sci. Rev., 86, 563, 1998.

Moen, J., Carlson, H. C., Milan, S., Shumilov, N., Lybekk, B., Sandholt, P. E., and Lester, M.: On the collocation between dayside auroral activity and coherent HF backscatter, Ann. Geophys., 18, 1531-1549, 2001a.

Moen, J., Holtet, J. A., Pedersen, A., Lybekk, B., Svenes, K., Oksavik, K., Denig, W. F., Lucek, E., and Andre, M.: Cluster boundary layer measurements and optical observations at magnetic conjugate sites, Ann. Geophys., 18, 1655-1668, 2001b.

Pinnock, M. and Rodger, A. S.: On determining the noon polar cap boundary from SuperDARN HF radar backscatter characteristics, Ann. Geophys., 18, 1523, 2001.

Rees, M. H.: Auroral ionization and excitation by incident energetic electrons, Planet. Space Sci., 11, 1209, 1963.

Rich, F. J. and Hairston, M.: Large-scale convection patterns observed by DMSP, J. Geophys. Res., 99, 3827, 1994.

Rodger, A. S., Mende, S. B., Rosenberg, T. J., and Baker, K. B.: Simultaneous optical and HF radar observations of the ionospheric cusp, Geophys. Res. Lett., 22, 2045, 1995.

Rodger, A. S., Coleman, I. J., and Pinnock, M.: Some comments on transient and steady-state reconnection at the dayside magnetopause, Geophys. Res. Lett., 27, 1359, 2000.

Russell, C. T., Snare, R. C., Means, J. D., Pierce, D., Dearborn, D., Larson, M., Barr, G., and Le, G.: The GGS/polar magnetic field investigation, Space Sci. Rev., 71, 563, 1995.

Russell, C. T., Wang, Y. L., and Raeder, J.: Possible dipole tilt dependence of dayside magnetic reconnection, Geophys. Res. Lett., 30(18), 1937, doi:10.1029/2003GL017725, 2003.

Sandholt, P. E. and Farrugia, C. J.: Monitoring magnetosheathmagnetosphere interconnection topology from the aurora, Ann. Geophys., 20, 629, 2002.

Sandholt, P. E., Deehr, C. S., Egeland, A., Lybekk, B., Viereck, R., and Romick, G. J.: Signatures in the dayside aurora of plasma transfer from the magnetosheath, J. Geophys. Res., 91, 10063 , 1986.

Sandholt, P. E., Farrugia, C. J., Stauning, P., Cowley, S. W. H., and Hansen, T.: Cusp/cleft auroral forms and activities in relation to ionospheric convection: Responses to specific changes in solar wind and interplanetary magnetic field conditions, J. Geophys. Res., 101, 5003, 1996.

Sandholt, P. E., Farrugia, C. J., Moen, J., Noraberg, O., Lybekk, B., Sten, T., and Hansen, T. L.: A classification of dayside auroral forms and activities as a function of IMF orientation. J. Geophys. Res., 103, 23 325, 1998.

Sandholt, P. E., Farrugia, C. J., Moen, J., and Denig, W. F. The cusp in rapid motion, J. Geophys. Res., 107(A12), 1427, doi:10.1029/2001JA009214, 2002.

Sandholt, P. E., Farrugia, C. J., and Denig, W. F.: Dayside aurora and the role of IMF $\left|B_{Y} / B_{Z}\right|$ : Detailed morphology and response to magnetopause reconnection, Ann. Geophys., 22, 613628, 2004.

Scudder, J. D.: Fluid signatures of rotational discontinuities, J. Geophys. Res., 89, 7431, 1984.

Scudder, J. D., Ogilvie, K., and Russell, C. T.: In magnetic reconnection in space plasmas, edited by Hones Jr., E. W., AGU Monograph 30, AGU, Washington D.C., 183, 1984.

Scudder, J. D., Hunsacker, F., Miller, G., Lobell, J., Zawistowski, T., Ogilvie, K., Keller, J., Chornay, D., Herrero, F., Fitzenreiter, R., Fairfield, D., Needell, J., Bodet, D., Googins, J., Kletzing, C., Torbert, R., Vandiver, J., Bentley, R., Fillius, W., McIlwain, C., 
Whipple, E., and Korth, A.: Hydra: A three dimensional electron and ion instrument for the polar spacecraft of the GGS mission, Space Sci. Rev., 71, 459-495, 1995.

Siscoe, G. L., Erickson, G. M., Sonnerup, B. U. Ö., Maynard, N. C., Siebert, K. D., Weimer, D. R., and White, W. W.: Magnetospheric sash dependence on IMF direction, Geophys. Res. Lett., 28, 1921-1924, 2001.

Smith, C. W., Acuña, M. H., Burlaga, L. F., L'Heureux, J., Ness N. F., and Scheifele, J.: The ACE magnetic field experiment, Space Sci. Rev., 86, 613, 1998.

Song, P., Russell, C. T., and Thomsen, M. F.: Slow mode transition in the frontside magnetosheath, J. Geophys. Res., 97, 8295, 1992.

Vorobjev, V. G., Gustafsson, G., Starkov, G. V., Feldstein, Y. I., and Shevnina, N. F.: Dynamics of day and night aurora during substorms, Plant. Space Sci., 23, 269, 1975.

Weimer, D. R.: An improved model of ionospheric electric potentials including substorm perturbations and application to the GEM 24 November 1996 event, J. Geophys. Res., 106, 407, 2000.
Weimer, D. R., Ober, D., Maynard, N. C., Burke, W. J., Collier, M. R., McComas, D. J., Ness, N. F., and Smith, C. W.: Variable time delays in the propagation of the interplanetary magnetic field, J. Geophys. Res., 107(A8), 10.1029/2001JA009102, 2002.

White, W. W., Siscoe, G. L., Erickson, G. M., Kaymaz, Z., Maynard, N. C., Siebert, K. D., Sonnerup, B. U. Ö., and Weimer, D. R.: The magnetospheric sash and cross-tail S, Geophys. Res. Lett., 25, 1605-1608, 1998.

White, W. W., Schoendorf, J. A., Siebert, K. D., Maynard, N. C., Weimer, D. R., Wilson, G. L., Sonnerup, B. U. Ö., Siscoe, G. L., and Erickson, G. M.: MHD simulation of magnetospheric transport at the mesoscale, in space weather, geophysical monograph series Vol. 125, edited by Paul Song, Howard J. Singer, and George L. Siscoe, pp. 229-240, Am. Geophys. Union, 2001.

Wing, S., Newell, P. T., and Ruohoniemi, J. M.: Double cusp: Model prediction and observational verification, J. Geophys. Res., 106, 25 571, 2001. 